\title{
General Relativity and Gravitation: A Centennial Perspective
}

\author{
Abhay Ashtekar ${ }^{1}$, Beverly K. Berger ${ }^{2}$, James Isenberg ${ }^{3}$, and Malcolm A. H. MacCallum ${ }^{4}$ \\ 1. Institute for Gravitation $\&$ the Cosmos, and Physics Department, \\ Penn State, University Park, PA 16802, U.S.A. \\ 2. 2131 Chateau PL, Livermore, CA 94550, USA \\ 3. Department of Mathematics, University of Oregon, Eugene, OR 97403-1222, USA \\ 4. School of Mathematical Sciences, Queen Mary University of London, \\ Mile End Road, London E1 4NS, U.K.
}

\begin{abstract}
To commemorate the 100th anniversary of general relativity, the International Society on General Relativity and Gravitation (ISGRG) commissioned a Centennial Volume, edited by the authors of this article. We jointly wrote introductions to the four Parts of the Volume which are collected here. Our goal is to provide a bird's eye view of the advances that have been made especially during the last 35 years, i.e., since the publication of volumes commemorating Einstein's 100th birthday. The article also serves as a brief preview of the 12 invited chapters that contain in-depth reviews of these advances. The volume will be published by Cambridge University Press and released in June 2015 at a Centennial conference sponsored by ISGRG and the Topical Group of Gravitation of the American Physical Society.
\end{abstract}

PACS numbers: 04.,95.30.,98.80.Jk

\section{INTRODUCTION}

The discovery of general relativity by Albert Einstein 100 years ago was quickly recognized as a supreme triumph of the human intellect. To paraphrase Hermann Weyl, wider expanses and greater depths were suddenly exposed to the searching eye of knowledge, regions of which there was not even an inkling. For 8 years, Einstein had been consumed by the tension between Newtonian gravity and the spacetime structure of special relativity. At first no one had an appreciation for his passion. Indeed, "as an older friend," Max Planck advised him against this pursuit, "for, in the first place you will not succeed, and even if you succeed, no one will believe you." Fortunately Einstein persisted and discovered a theory that represents an unprecedented combination of mathematical elegance, conceptual depth and observational success. For over 25 centuries before this discovery, spacetime had been a stage on which the dynamics of matter unfolded. Suddenly the stage joined the troupe of actors. In subsequent decades new aspects of this revolutionary paradigm continued to emerge. It was found that the entire universe is undergoing an expansion. Spacetime regions can get so warped that even light can be trapped in them. Ripples of spacetime curvature can carry detailed imprints of cosmic explosions in the distant reaches of the universe. A century has now passed since Einstein's discovery and yet every researcher who studies general relativity in a serious manner continues to be enchanted by its magic.

The International Society on General Relativity and Gravitation commissioned a volume to celebrate a century of successive triumphs of general relativity as it expanded its scientific reach. It contains 12 Chapters, divided into four Parts, highlighting the advances that have occurred during the last 3-4 decades, roughly since the publication of the 1979 
volumes celebrating the centennial of Einstein's birth. During this period, general relativity and gravitational science have moved steadily toward the center of physics, astrophysics and cosmology, and have also contributed to major advances in geometrical analysis, computational science, quantum physics and several areas of technology. The next two decades should be even more exciting as new observations from gravitational wave detectors and astronomical missions open unforeseen vistas in our understanding of the cosmos. The volume provides a vivid record of this voyage.

The organization of the volume is as follows:

PART I: EINSTEIN'S TRIUMPH

Chapter 1:

100 Years of General Relativity

George F. R. Ellis

Chapter 2:

Was Einstein Right?

Clifford Will

Chapter 3:

Relativistic Astrophysics

John Friedman, Peter Schneider, Ramesh Narayan, Jeffrey E. McClintock, Peter Mészáros, and Martin Rees

Chapter 4:

Cosmology

David Wands, Misao Sasaki, Eiichiro Kamatsu, Roy Maartens and Malcolm A. H. MacCallum

PART II: NEW WINDOW ON THE UNIVERSE

Chapter 5:

Receiving Gravitational Waves

Beverly K. Berger, Karsten Danzmann, Gabriela Gonzalez, Andrea Lommen, Guido Mueller, Albrecht Ruediger, and William Joseph Weber

Chapter 6:

Sources of Gravitational Waves: Theory and Observations

Alessandra Buonanno and B. Sathyaprakash

PART III: GRAVITY IS GEOMETRY AFTER ALL

Chapter 7:

Probing Strong Field Gravity Through Numerical Simulations

Frans Pretorius, Mattew Choptuik and Luis Lehner

Chapter 8:

Initial Data and the Einstein Constraint Equations

Gregory Galloway, Pengzi Miao and Richard Schoen

Chapter 9:

Global Behavior of Solutions to Einstein's Equations

Stefanos Aretakis, James Isenberg, Vincent Moncrief and Igor Rodnianski 
PART IV: BEYOND EINSTEIN

Chapter 10:

Quantum Fields in Curved Space-times

Stefan Hollands and Robert Wald

Chapter 11:

From General Relativity to Quantum Gravity

Abhay Ashtekar, Martin Reuter and Carlo Rovelli

Chapter 12:

Quantum Gravity via Supersymmetry and Holography

Henriette Elvang and Gary Horowitz

The goal of these Chapters is two-fold. First, beginning researchers should be able to use them as introductions to various areas of gravitational science. Second, more advanced researchers should be able to use the Chapters that are outside the area of their immediate expertise as overviews of the current status of those subjects. Since the scope of gravitational science has widened considerably in recent years, the volume should be useful not only to specialists in general relativity but also to researchers in related areas.

In this article we have collected the detailed introductions to the four Parts. They summarize the main advances and offer a short tour of the more detailed material that can be found in the Chapters that follow. The introductions also contain illustrative examples of outstanding important open issues, and outline important developments that could not be included in individual Chapters where the authors had the difficult task of covering many developments in a limited space. We hope that this material will provide a global perspective on developments in the four main areas of gravitational science.

\section{PART I: EINSTEIN'S TRIUMPH}

Recent media attention to the centenary of the outbreak of the First World War (WWI) reminds us that it was against this backdrop that Einstein, a Swiss citizen, announced the revolutionary theory of general relativity (GR). The war affected the theory's dissemination. Eddington's report introducing GR to the English-speaking world [1] relied on information from de Sitter in neutral Holland. Inevitably, the theory's adherents were caught up in the conflict, most notably Karl Schwarzschild, who died in 1916 while serving on the Russian front.

In 1915 Einstein was already a decade on from his annus mirabilis of 1905, in which he had announced the theory of special relativity, explained the already well-observed photoelectric effect as due to quantization of light (a vital step towards quantum theory), and explained Brownian motion assuming the reality of atoms, an explanation experimentally confirmed by Perrin in 1908. The second of these three great papers won him the 1921 Nobel prize - and they were not all he published that year! For example, he gave the famous $E=m c^{2}$ equation, which later gave the basis of nuclear fusion and fission (whence Einstein's intervention in the development of atom bombs). Fusion in particular explained how stars could hold themselves up against gravity as long as they do. So Einstein had already triumphed well before 1915 .

However, he was aware that his work left an awkwardly unresolved question - the need for a theory of gravity compatible with special relativity that agreed with Newton's theory in an appropriate limit. Here we will not recount Einstein's intellectual development of 
general relativity, which resolved that problem, nor describe the interactions with friends and colleagues which helped him find the right formulation. Those are covered by some good histories of science, and biographies of Einstein, as well as his own writings.

The theory's prediction of light-bending confirmed to good accuracy [2] by the UK's 1919 eclipse expedition led by Eddington ${ }^{1}$ and Crommelin, brought Einstein to the attention of the general public, in particular through the famous headline in the New York Times of November 9th. From then on, he increasingly came to be seen as the personification of scientific genius.

Why then are we calling this first Part of our centennial book "Einstein's triumph"? GR had already triumphed by 1919 .

The triumph since 1919 lies in GR's ever increasing relevance and importance, shown in particular by the number and range of applications to real world observations and applications, from terrestrial use in satellite navigation systems to considerations of cosmology on the largest scales. Moreover the different applications are now interwoven, for example in the relevance of black holes in cosmology and the use of pulsars and compact relativistic stars in strong field tests of the theory. This Part of the book outlines that progress.

As Ellis describes in Chapter 1, the starting points for many later confirmations were laid in the early years of the theory: the Schwarzschild solution, leading to solar system tests and black hole theory; light-bending, which grew into gravitational lensing; and the Friedmann(Lemaître-Robertson-Walker: FLRW) solutions, basic in cosmology. Moreover, several confirmations relate to the three "classical tests": gravitational redshift, the anomaly in the perihelion advance of Mercury as computed from Newtonian theory ${ }^{2}$, and light-bending: for example, the analysis of GPS (the Global Positioning System), the study of the binary and double pulsars, and the use of microlensing to detect exoplanets. The theory remains the most nonlinear of the theories of physics, prompting development in analytic and numerical technique.

Classical differential geometry as studied in introductory courses (and as briefly outlined by Ellis) is adequate to discuss the starting points of those developments. But they soon require also the proper understanding of global structure and thus of singularities and asymptotics, for example in understanding the Schwarzschild solution, black holes and the energy carried away by gravitational radiation. This increasing sophistication was reflected in the best-selling text of Hawking and Ellis [3], and further developments are described in Part Three of this book.

Much of the development of GR has come in the last half century. For its first 50 years, a time when quantum theory was making big advances, one could argue that GR remained an intellectual ornament with only some limited applications in astronomy. Even its relevance to cosmology was debatable, because Hubble's erroneous distance scale led to a conflict between the geologically known age of the Earth and the age of the universe in a FLRW model, prompting the range of alternative explanations for this discrepancy described in Bondi's book [4]. While the notion of a stagnant phase is rather belied by the many significant papers from this time which have deservedly been included in the "Golden Oldies" series of the General Relativity and Gravitation journal, some of them cited by Ellis, it was certainly a less dynamic period than the following 50 years of GR.

\footnotetext{
${ }^{1}$ How Eddington, a Quaker, while preparing for this expedition, avoided being sent to work on the land as a conscientious objector, is itself an interesting WWI story.

${ }^{2}$ One may note that the anomalous part is $43^{\prime \prime}$ per century in a total of around $5000^{\prime \prime}$ per century.
} 
The changes have been partly due to the already mentioned increasing mathematical sophistication among theoretical physicists. Taub's use of symmetry groups [5], and Petrov's algebraic classification of the Weyl tensor [6] were crucial steps forward made in the 1950s. The geometric concepts of connection and curvature have become fundamental in modern gauge theories. Progress in the theory of differential equations has given a firm basis to the idea that GR is like other physical theories in that initial configuration and motion determine the future evolution. The generating techniques for stationary axisymmetric systems used to obtain exact solutions ${ }^{3}$ relate to modern work on integrable systems. Further developments in such areas are reflected in Chapter 1 and Part Three of this book.

Another important step was introducing the theory of the matter content within FLRW models. This enabled the understanding of the formation of the chemical elements, by combining the Big Bang and stellar nucleosyntheses, the provision of evidence that there were only three types of neutrino, and the prediction of the Cosmic Microwave Background (CMB).

Progress has depended even more on advances in technology and measurement technique. The first example was the revision of Hubble's distance scale in 1952 by Baade, using the 200 inch Palomar telescope commissioned in 1950. This led to increasing belief in the FLRW models, a belief eventually cemented by the 1965 observations of the CMB, which themselves arose from developments in microwave communications technology.

The 1957 launch of the first artificial satellite, Sputnik, intensified the need for detailed calculation of orbital effects in satellite motion, in order to very accurately plan satellite projects. Such work [9] was undertaken for both the US and USSR programs and was the first practical use of GR.

Radio astronomy, by showing source counts inconsistent with the alternative Steady State theory, had provided important evidence for FLRW models. It also, combined with optical observations, led to the discovery of quasars ${ }^{4}$ which prompted Lynden-Bell to propose that they were powered by black holes [10]: the importance black holes have subsequently assumed in our understanding of astronomy and cosmology is described by Narayan and McClintock in Chapter 3. Radio astronomy also discovered the pulsars, announced in 1968, which gave extra impetus to the already developing study of relativistic stars, discussed by Friedman in Chapter 3.

The reality of gravitational waves in the theory, which had been debated earlier, was finally clarified in the work of Bondi et al in 1959 [11]. The binary and double pulsar observations, described in Chapter 2, united the understanding of compact objects and gravitational waves to provide the first strong field tests of GR.

The exquisite precision now achieved in practical and observational areas of GR has made use of the development of very high precision atomic clocks and of the burgeoning of electronics since the invention of the transistor in 1947. Satellite-borne telescopes in several wavebands, computers of all scales from the largest (used in numerical relativity) to mobile devices (e.g. in GPS receivers), CCD devices (based of course on the photoelectric effect), and lasers (in terrestrial gravitational wave detectors - also used, for example, in determining the exact position of the moon) have all played major roles in the observations

\footnotetext{
${ }^{3}$ The construction and interpretation of exact solutions are topics not covered by this book, as they are well covered by [7] and [8] and references therein. In particular we do not consider some important techniques used in those areas, such as computer algebra and the application of local spacetime invariants.

${ }^{4}$ 3C48 was identified in 1960 and 3C273's redshift was found in 1963.
} 
and experiments described in the following four Chapters (and in the later parts of the book).

There were fundamental aspects of gravity (e.g. the Eötvös effect) which could be and were tested on Earth, but until the 1970s the focus was on the "classical tests", complemented by the time delay measurements for satellites. Dicke initiated a more systematic analysis of the equivalence principle and its tests, as described in Chapter 2. Thorne, Will and others then developed other frameworks, notably the PPN framework, which could encompass other types of tests. While the application of these ideas still relied on solar system and terrestrial tests, these became much more precise and involved much new technology (e.g. laser ranging to the moon, superconducting gravimeters on the ground, use of atomic traps and atomic clocks in terrestrial and satellite experiments), and pinned the parameters of the PPN framework down with high precision.

Tests outside the solar system consisted of the understanding of compact stars such as white dwarfs, and supernova remnants, and of cosmology (for which there was only an incomplete understanding, for reasons described below), but did not lead to new precise constraints on the theory. That changed with the discovery and observations of the (first) binary pulsar, and still further with the several now known, including the double pulsar. These give some of the most precise measurements in physics (although, perhaps surprisingly, the Newtonian constant of gravitation, $G$, remains the least accurately known of the fundamental constants of nature).

It is notable that the understanding of pulsars not only required GR (because of the strong fields) but also entailed the simultaneous use of quantum theory and GR (because only by taking into account quantum theory could one have adequate equations of state to model white dwarfs and neutron stars). These types of compact objects, and black holes, are now the starting points for the calculation of gravitational wave sources described in Part Two.

Relativistic astrophysics then developed in a number of directions (see Chapter 3). Numerical simulations gave much more detail on relativistic stars, their properties, stability and evolution. A whole new sub-discipline of black hole astrophysics came into being, concerned with the environments of black holes, especially (for stellar size black holes) accretion from neighboring stars, and (for supermassive black holes) accretion, nearby orbits and tidal capture of stars. The improved understanding enabled us to be rather certain not only that there really are black holes in the Universe, but that they are very common.

A further direction described in Chapter 3 came about with the discovery and increasingly detailed observations of gamma ray bursts. Both their long and short varieties turned out to require models of relativistic sources, as described by Mészáros and Rees. It is interesting that there is a link with the gravitational wave detectors described in Part Two, in that the absence of gravitational waves from GRB 070201 showed that, if it had a compact binary progenitor, then that progenitor had to be behind rather than in M31 [12].

While the standard FLRW models used up to 1980 or so did very well in describing the observed isotropy and homogeneity of the universe, and explaining the evolution of the matter content which led to formation of the chemical elements and the prediction of the CMB, they failed to explain the single most obvious fact about the Universe, namely that it has a highly non-uniform density. Naturally occurring thermal fluctuations and their evolution could not give large enough variations. The inflationary paradigm, introduced by Guth in 1981 [13], altered that radically by providing reasons for a nearly flat spectrum of density fluctuations at a time sufficiently early in the universe for the subsequent linear 
and nonlinear phases of evolution to produce the observed structures we see. The theory is described in detail by Sasaki in Chapter 4.

The resulting standard model has been compared with a range of very high precision observations, notably those of the $\mathrm{CMB}$, the baryon acoustic oscillations (BAO) and the magnitude-redshift relation for supernovae (relating distances and expansion velocities in the Universe). These, especially the CMB observations, have generated the title "precision cosmology", which, as Komatsu emphasizes in Chapter 4, requires precision theory as well as precision observation. That precision in theory consists of very detailed consideration of perturbations of the FLRW models and of light propagation in perturbed models, enabling the link between the conditions produced by inflation (or some alternative to inflation providing suitable initial conditions) and the present-day observations. What is remarkable is the fine detail of those initial conditions that one can infer from observation.

To some degree, the role of GR has disappeared in the large volume of literature related to CMB, BAO and supernova, and other observations, as almost all of it uses the FLRW models and their linearized perturbations, and may even use Newtonian analyses in key steps. Wands and Maartens remind us, in their introduction to Chapter 4, that GR in fact still has a crucial role to play, even in precision cosmology where its effects may be considerably larger than the very small error bars in the observations, and the correlations described in Chapter 3 imply it also has a role to play in structure formation below the scales tested by the CMB. Moreover it is essential in testing the robustness of the assumptions of the concordance model, a further topic discussed in Chapter 4.

What can we expect in the future? The results of Planck's B-mode polarization ${ }^{5}$ measurements, confirming or contradicting those of BICEP2 and POLARBEAR (both of these being outlined in Chapter 4), may be published before this book. In 2015 Advanced LIGO will begin taking data (see Part Two). If such advanced gravitational wave detectors see the expected gravitational wave sources, we will have a new window for testing GR (but if no such sources are seen, that may be due only to poor astrophysical predictions). In the past, when new windows on the universe have opened, new and unforeseen phenomena have been found [14]; it would not be surprising if this happens again. Beyond that there are a plethora of new instruments being built or planned to study the sky in electromagnetic wavebands from low frequency radio to $\gamma$-rays: the chances of convincing funding agencies to support such work have probably been substantially enhanced by the spectacular results of recent past projects.

Gravitational lensing by galaxies seemed to surprise many when first found in 1979, even if it should not have. Now such lensing, along with its stellar size counterpart, have become tools for astronomy, used for example to infer the distribution of mass within galaxies, the distribution of dark matter, the properties of distant galaxies, and the presence of new exoplanets. Recently, magnification due to microlensing was used to determine properties of a binary system containing a white dwarf and a Sun-like star [15].

We stress again that the galactic and intergalactic application is just one of the instances where different aspects of GR come together - here lensing and cosmological models.

Although the greatest challenge for GR may lie in finding and testing a good enough

\footnotetext{
${ }^{5}$ There are two characteristic patterns of polarization alignments expected in the CMB. The E-mode is like that of the electric field round a charge and the B-mode like that of magnetic field round a current. Instances of these modes, with varying amplitudes and centered at random locations, will be superposed in the actual observations. For more details see Chapter 4.
} 
theory of quantum gravity, as discussed in Part Four, there are still challenges at the classical level. Cosmology provides the greatest of these, since its standard model requires three forms of matter - the inflaton, dark matter and dark energy - which have not been, and perhaps cannot be, observed in terrestrial laboratories, and whose properties are modeled only in simple and incomplete ways. It would of course be ironic if the triumph of GR in cosmology were to turn to disaster because the only way to deal with those apparently-required three forms of matter were to adopt a modified theory of gravity, but other explanations seem much more likely.

The inflaton is postulated as a way to produce the nearly flat spectrum of fluctuations required as initial data from which acoustic oscillations produce the observed CMB power spectrum. While the assumptions of inflation may be questionable, it is, as already mentioned, remarkably successful in producing the right distribution of fluctuations on present day scales above $150 \mathrm{Mpc}$ or so (a scale much larger than that of individual galaxies). Moreover the recent detection of B-modes claimed by BICEP2 is consistent with inflation theory's prediction of polarization due to gravitational waves. A definitive detection of such polarization would provide indirect evidence on quantum gravity and the quantum/classical correspondence, in that the theory assumes the quantum fluctuations of the inflationary era become classical.

The evidence for dark matter is rather securely based on observations at scales where a Newtonian approximation is good enough to show that not all the mass is visible, such as observations of galactic rotation curves and the distribution of X-ray emitting gas in clusters. It provides $25-30 \%$ of the critical energy density of the Universe, itself now known to be very close to the actual energy density (see Chapter 4). This was known before the more precise $\mathrm{CMB}$ and baryon acoustic oscillation (BAO) measurements [16]. Additional evidence for dark matter has been provided by comparing the distribution of mass in colliding galaxies, as shown by its lensing effects, with the mass distribution of the visible gas. However, a change in the gravity theory might provide an explanation for these observations not requiring dark matter, though as yet no satisfactory such theory has been proposed.

The inference of the existence of dark energy is even more dependent on GR, in particular on the theory of perturbed FLRW models (see Chapter 4): it comes from the magnituderedshift relation for supernovae (relating distance and expansion velocity of the Universe), $\mathrm{CMB}$ and BAO data. Attempted explanations within GR not requiring a new form of matter (in which we include the cosmological constant) have used both large and small scale inhomogeneities (see Chapter 4), or may arise from the astrophysics of supernovae and their environments. Or we may be able to pin down the properties of dark energy in some way independent of FLRW models, and thereby provide a further triumph for the predictions of GR.

Obtaining information about the three so far unobserved constituents of the standard model may not come from GR itself. But we would certainly like a better understanding of inhomogeneities and their back reaction and impact on light propagation. The evidence of correlations of galactic properties with central black hole masses suggests we also need to know much more about the messy non-linear processes of galaxy and star formation and their interaction with the nonlinearities of GR.

Despite these lacunae, which may offer opportunities for future breakthroughs, when taken together the following four Chapters illustrate very well the staggering extent of the triumph of Einstein's 1915 proposal of the theory of General Relativity. 


\section{PART II: NEW WINDOW ON THE UNIVERSE}

Gravitational waves provide an opportunity to observe the universe in a completely new way but also give rise to an enormous challenge to take advantage of this opportunity. When Einstein first found wave solutions in linearized general relativity and derived the quadrupole formula, it became clear that a laboratory experiment to produce and detect gravitational waves was impossible while it was also clear that any gravitational wave signals produced astronomically were too weak to be detected on earth with the instruments available or thought possible at that time. Nearly 100 years later, we are at the confluence of fundamental science and technology that will soon open this new window.

Several lines of development were required to make the search for gravitational waves realistic. Despite the early recognition by Einstein that linearized gravity had wave solutions, the physical reality of gravitational waves remained in dispute for many decades. The reason for this was the absence of formalisms able to separate physical degrees of freedom in the field equations from coordinate (gauge) effects. A well known, striking example was Einstein's conviction that the Einstein-Rosen cylindrical waves [17] were not physical and furthermore that the character of this exact solution proved that there were no physical gravitational waves in the full theory. While Einstein retrieved the correct interpretation in the nick of time [18], the question remained unsettled until correct, gauge invariant formulations of the problem were developed. The first of these, from Bondi's group [19 21], used the "news function" to demonstrate that, far from the source, one could quantify the energy carried away by gravitational waves. Further developments in understanding equations of motion, gauge freedom, and other methods to identify gravitational waves in the background spacetime led to approximation methods with greater precision and broader application than the original linear waves [22, 23]. In addition, the first half-century of general relativity saw the physically relevant exact solutions of Schwarzschild [24] and, much later, Kerr [25]. In the late 1930s, Oppenheimer and Volkoff [26] and Oppenheimer and Snyder [27] studied spherically symmetric gravitational collapse that predicted the formation of neutron stars and black holes respectively even though both categories were considered highly unlikely as real objects for several decades thereafter.

Meanwhile, astronomical technology developed beyond the optical frequency band. Radio astronomy coupled to optical followup led to the discoveries of quasars in 1963 and pulsars in 1967 to usher in the age of relativistic astrophysics - not to mention the discovery of the cosmic microwave background (CMB) in 1965. Suddenly, the universe became much more interesting for general relativity. X-ray detectors aboard rockets found bright point sources outside the solar system, possibly indicating a more violent universe than previously suspected. Supernovae could now be better studied and served as the driver for early general relativistic computer simulations [28]. At approximately the same time as physical systems in the strong-gravity regime became plausible, theoretical developments made it clear that gravitational waves could carry energy and thus could be physically relevant. At some point, improved treatment of the two-body problem in general relativity led to a number of recalculations of Einstein's quadrupole formula, engendering a rather vitriolic and long reigning dispute over the coefficient. It became clear later (in the 1980s) that consistent approximations yielded Einstein's results [29].

The possibility that detectable gravitational wave sources might exist and the firm theoretical foundation that such waves were a prediction of general relativity inspired Joseph Weber to build a detector to search for them [30]. His first detector, an aluminum cylinder 
(bar) with piezoelectric readout is described in [31, 32]. He quickly realized that coincident detection - i.e., two or more bars - was an essential ingredient. This first generation of bar detectors reached a sensitivity to gravitational wave strain amplitudes of $10^{-16}$ by the end of the 1960s [33]. Weber's electrifying claim of the detection of signals associated with the center of our galaxy [32] inspired a worldwide effort leading to one or more bar detectors operating in China, France, Germany, Italy, Japan, UK, USA, and USSR [33, 34]. Unfortunately, even though the subsequent experiments of this first generation of bars were more sensitive than Weber's, they failed to confirm his detection. In addition to bar development, plans were made to use the earth [35], the moon [36], and a number of spacecraft [37] as gravitational wave detectors. By the first decade of this century, the largest bars had reached the size of 3 meters and the mass of more than 2 tons, were operated at cryogenic temperatures as low as $0.1 \mathrm{~K}$ to minimize thermal noise, and achieved a strain sensitivity of $\approx 10^{-21} / \sqrt{\mathrm{Hz}}$ at $900 \mathrm{~Hz}$. Prototypes of novel designs for resonant mass detectors were developed in this period with at least one prototype currently under construction [34].

In the mid 1970s, interferometric detectors were first considered both for the ground and for space. Chapter 5 focuses on highlights of the current status of such instruments. The first prototypes were built by Forward (at Hughes) and later by Weiss (at MIT) and Drever (at Glasgow and Caltech) as well as elsewhere in the world [33]. The laboratory-scale interferometers had sensitivities far below those of the contemporaneous bars. However, the interferometers could be scaled up in contrast to bars (see [38]) but would require facilityclass dimensions and funding to achieve sensitivities that were plausible for detection.

We note here that gravitational wave detection pushes precision technology and related theory and thus can yield important scientific spinoffs. An early example is quantum nondemolition. The broad idea is to overcome the standard quantum limit by arranging to decrease the uncertainty in one variable by increasing it in the complementary variable. This was first proposed for bar detectors by Braginsky et al [39] and refined for interferometers by Caves [40]. The concept has been studied extensively in atomic, molecular, and optical physics since then but is most spectacularly implemented on the kilometer-scale interferometer GEO 600 [41] and was tested successfully on one of the LIGO instruments [42]. It is likely to become a near-term upgrade for the second generation interferometers Advanced LIGO and Advanced Virgo.

Meanwhile, in the early 1970s, the Uhuru X-ray satellite revealed the X-ray sky for the first time (where brief rocket flights had only given hints). Stellar mass binaries, including Cyg X-1, a strong black hole candidate, as well as a number of active galactic nuclei, were revealed and could be correlated with radio and optical sources. It became possible to argue that potential gravitational wave sources were abundant.

This time period also saw the first steps toward binary black hole simulations by Smarr and collaborators [43]. However, the landmark discovery of this decade was the binary pulsar PSR1913+16 by Hulse and Taylor [44]. Not only was this the first known system containing two neutron stars, but the orbital parameters made it sufficiently general-relativistic to strongly constrain theories of gravity (see the discussion in Chapter 2). Even more exciting, the period decay due to energy loss by emission of gravitational waves was measurable. Because this system is clean (no extraneous effects from tides or matter accretion), it provided incontrovertible experimental evidence of the existence of gravitational waves and also of the correctness of the quadrupole formula (see the discussion in Chapter 6). As a side note, this validation of the quadrupole formula killed off the last vestiges of debate about the coefficient. In addition, the known binary neutron star systems are precursors of gravitational 
wave sources for ground-based detectors - and, in fact, are the only ones that demonstrably exist. Although supernovae in our galaxy may be detectable sources, the details of nonaxisymmetric collapse are not yet known sufficiently well to predict their strength. What this means is that the orbit decay of binary neutron stars will eventually lead to the final inspiral and merger phase that produces strong gravitational waves at frequencies of up to approximately one $\mathrm{kHz}$ (see Chapter 6). Because the time to merger for the known systems is significantly less than the age of the universe, one has reason to hope that such systems in the merger phase occur with sufficient frequency to be a target for human detection if sufficiently sensitive instruments could be built. As more (but still a small number) such systems were discovered and stellar evolution became better understood, event rate estimates became possible [45]. Chapter 6 focuses on the gravitational-wave signatures of these and other proposed sources. To obtain the correct waveform requires either careful (or even rigorous) approximations or robust numerical simulations.

While the best understood source for ground-based interferometric detectors has been observed electromagnetically in its precursor phase, actual known, named objects, namely white dwarf binaries, are accessible to space-based detectors which when constructed and launched will operate at the much lower frequencies characterizing such systems. Examples are given in Chapter 6.

A byproduct of the expansion of astronomy into non-optical detection was the identification of systems of stellar mass and up to $10^{9} \mathrm{M}_{\text {sun }}$ which could be black holes. Over the years, the black hole hypothesis has become the favored explanation for these systems. Black hole binary systems, if they exist, would be an obvious target for gravitational wave detection since in a clean environment of no accretion discs they would emit no electromagnetic signals. Hawking had proved that a merging binary black hole could emit up to $29 \%$ of its mass-energy in gravitational waves [46]. But what was the actual number? To answer this question required numerical simulations in three spatial dimensions (3D). While the 2D (head on collision) problem was solved reasonably well by Smarr and collaborators [43] in the 1970 s, the 3D problem proved astonishingly difficult until it was finally solved in 2005 [47-49] (see Chapter 7).

The growth of relativistic astrophysics made the scientific case for development of gravitational wave detection programs compelling. Although many bar groups left the field after their failure to confirm Weber's claims, several remained and continued to improve their instruments [33]. Nonetheless, though the interferometric prototypes built in the 1970s and 1980s were less sensitive than the bars of their era, it became clear that interferometers were the only way to achieve the needed sensitivity in the frequency range accessible from the ground. Efforts were begun in the US, Germany, UK, France, Italy, and Japan to seek funding to build the kilometer-scale instruments that would be necessary to detect a strong gravitational wave event from the Virgo cluster (see Chapter 5). This distance was chosen as a criterion because it is necessary to probe at least that far to have sufficient likelihood for a detectable event (see Chapter 6). Remarkably, given the novelty and scale (both physically and financially) of the proposed technology, two kilometer-scale projects, LIGO in the US and Virgo, a French/Italian collaboration in Italy, with $4 \mathrm{~km}$ and $3 \mathrm{~km}$ arm-lengths respectively, were funded and built. Construction took place in the 1990s and data taking by some of the instruments, as well as by the shorter but nearly kilometer-scale GEO 600 and TAMA, began in about 2000 (see Chapter 5).

Meanwhile, building on earlier efforts with data from prototype detectors [50], programs to analyze the data coming from LIGO, Virgo, GEO 600, and TAMA were initiated. The 
time series $h(t)$ for the gravitational wave strain was analyzed either by matched filtering (comparing to templates) for binary neutron stars or as unmodeled bursts (see Chapters 5 and 6). Note that the former required the substantial computational power provided by advancing technology to become feasible. Toward the end of the 2000s, astrophysically interesting upper limits were obtained, the technological tour-de-force of design sensitivity was achieved, and bar sensitivity was exceeded [51].

In 2010, an upper limit for burst events was published, based on observations by a worldwide network of bars [52]. While the limit itself was not interesting astrophysically, the effort demonstrated the advantages of international collaboration in this field. In addition, the technology to rotate the LSU bar was developed and implemented. This is interesting because a cross-correlation, stochastic measurement with the LIGO Livingston instrument allowed regular reorientation of the bar to impose a periodicity on any signal present since the allowed orientations included a direction where there should be no correlation [53].

Starting in the 1980s, space-based concepts were proposed and evaluated leading to adoption of LISA (first envisioned by Bender and Faller) as a joint R\&D project of NASA and ESA in 1998. The astronomical community became engaged, possibly due to the existence of the white-dwarf-binary calibration sources. Regrettably, in contrast to the ground-based detectors supported through physics programs, the astronomy-centered space programs in Europe and the US never moved LISA into a launchable status. One issue was the alleged riskiness of the technology. While the level of precision needed for LISA is six orders of magnitude less than for, e.g., LIGO due to LISA's proposed arm-lengths of millions of kilometers (see Chapter 5), the picometer level of precision required is still daunting for space-qualified missions and several necessary technologies are new. It is hoped that the often postponed but now firmly scheduled LISA Pathfinder will demonstrate the feasibility of LISA and LISA-like missions (see Chapter 5).

In the past decade or so, increasing interest has arisen in taking advantage of the remarkable timing precision and stability associated with millisecond pulsars to use the monitoring of pulse arrival times to tease out signals from passing gravitational waves [54]. These methods are effective at nanohertz frequencies and are sensitive to signals from supermassive binary black holes and from a stochastic background. In principle, the ability to detect a gravitational wave signal increases with increasing monitoring time (with a power that depends on the type of source). At this time, it is not clear that the systematic errors are completely understood. Nevertheless, there is a significant chance that pulsar timing arrays will detect gravitational waves within this decade (see Chapter 5).

Of course, we should not leave out the imprint of gravitational waves on the cosmic microwave background (see Chapter 3). In particular, at certain angular scales, relic gravitational waves produced in the early universe leave a curl-like tensor imprint, the so-called B-modes, on the cosmic microwave background. If the signal is sufficiently strong, with the ratio of tensor-to-scalar polarization amplitude $r$ large enough to be currently observable, the gravitational waves are likely to have been produced by cosmological graviton production and amplified by inflation. A recent report of such a detection with $r \approx .2$ by BICEP2 may have been premature since the measured effect could be caused by foreground dust or have a significant dust component [55]. Whether or not this possible detection of gravitational waves is confirmed, the discussion centered on the observation emphasizes that direct or indirect detections of gravitational waves reveal information about the universe and its contents that are inaccessible with electromagnetic radiation, cosmic rays, or even neutrinos.

A number of different technologies have been applied to the different programs for gravi- 
tational wave detection. These are not in competition but rather are complementary in that they search for gravitational waves in different frequency bands. As with electromagnetic emitters, gravitational waves at different frequencies arise from different sources. In simple terms, the frequency depends inversely on a power of the mass (see Chapter 6). At the highest frequencies of $10 \mathrm{~Hz}-10^{4} \mathrm{~Hz}$ accessible from the ground, the predominant sources are expected to be "chirp signals" from binary neutron stars or binary black holes with masses of order 1-300 $\mathrm{M}_{\text {sun }}$, "monochromatic" signals from non-axisymmetric rotating neutron stars, correlations between the noise from two detectors that characterize a cosmological or astrophysical stochastic background, or unmodeled bursts from supernovae in our galaxy or other sources. The ground-based detectors certainly have the potential to identify the engines of gamma ray bursts [56]. Future generations may be able to probe the equation of state of nuclear matter, discover intermediate mass black hole binaries, and reveal as yet unsuspected new phenomena. Space-based observations are sensitive to binary white dwarfs and binary neutron stars in the early inspiral phase and binary black holes in the mass range of $10^{5}$ to $10^{7} \mathrm{M}_{\text {sun }}$. Perhaps more interestingly for general relativity, they can observe the inspiral of a stellar mass black hole around a supermassive black hole. If the gravitational waves from such sources can be observed with sufficient precision [56], detailed tests of general relativity can be made. One is the definitive "smoking gun" detection showing that the central object is a black hole (see Chapter 6). Another allows one to obtain an interesting bound on the graviton mass through a distortion of the phasing of gravitational waves in a binary black hole inspiral [57]. Finally, pulsar timing arrays can search for gravitational wave signals from supermassive binary black holes that are suspected to exist from their electromagnetic properties, as well as ones that are so far unsuspected. Once gravitational waves are detected, it would be natural to expect that the experience of the early radio, X-ray, and $\gamma$-ray telescopes will be repeated with the discovery of completely new and unexpected phenomena to, once again, widen our horizons in unforeseen directions.

The next two chapters explore the sources and technologies that comprise gravitational wave science. They emphasize the growth in our knowledge about the sources through observations, theory, and simulations, including the state of our knowledge on their numbers (or number density) in the universe, and their gravitational wave signatures. These are then the targets for decades of technological development for detectors on earth and in space with the former on the verge of recording their first signals.

When looking back over a century of general relativity, we see that gravitational waves not only represent a future transformative discovery but have also, over the past 30 years or so, changed the nature of the field of gravitational physics. General relativity has gone from a discipline with few practitioners exploring apparently arcane (but often seminal) mathematical properties of the theory to big science with international collaborations yielding publications with nearly 1000 authors whose instruments require an investment on the order of $\$ 1$ billion (US). A further transformation will occur with the first detections by the second generation Advanced LIGO and Advanced Virgo instruments, independent of the precise nature of the sources. Unlike the cosmic microwave background measurements or pulsar timing arrays that piggyback on instruments developed for other purposes, the instrumentation developed for gravitational-wave detection on the ground and in space represents a completely new way to study the universe. It is likely that the discoveries soon to be made by these advanced detectors will "shake loose" the future of the field by accelerating the timeline for the first space-based gravitational wave detectors and by encouraging investment in third generation instruments such as the proposed Einstein Telescope [58]. 


\section{PART III: GRAVITY IS GEOMETRY, AFTER ALL}

Einstein's general relativity is a mathematically beautiful application of geometric ideas to gravitational physics. Motion is determined by geodesics in spacetime, tidal effects between physical bodies can be read directly from the curvature of that spacetime, and the curvature is closely tied to matter and its motion in spacetime. When proposed in 1915, general relativity was a completely new way to think about physical phenomena, based on the geometry of curved spacetimes that was largely unknown to physicists.

While the geometric nature of Einstein's theory is beautiful and conceptually simple, the fundamental working structure of the theory as a system of partial differential equations (PDEs) is much more complex. Einstein's equations are not easily categorized as wave-like or potential-like or heat-like, and they are pervasively nonlinear. Hence, despite the great interest in general relativity, mathematical progress in studying Einstein's equations (beyond the discovery of a small collection of explicit solutions with lots of symmetry) was quite slow for a number of years.

This changed significantly in the 1950s with the appearance of Choquet-Bruhat's proof that the Einstein equations can be treated as a well-posed Cauchy problem [59]. The long term effects of this work have been profound: Mathematically, it has led to the present status of Einstein's equations as one of the most interesting and important systems in PDE theory and in geometrical analysis. Physically, the well-posedness of the Cauchy problem for the Einstein equations has led directly to our present ability to numerically simulate (with remarkable accuracy) solutions of these equations which model a wide range of novel phenomena in the strong field regime.

The Cauchy formulation of general relativity splits the problem of solving Einstein's equations, and studying the behavior of these solutions, into two equally important tasks: First, one finds an initial data set-a "snapshot" of the gravitational field and its rate of change - which satisfies the Einstein constraint equations, which are essentially four of the ten Einstein field equations. Then, using the rest of the equations, one evolves the gravitational fields forward and backward in time, thereby obtaining the spacetime and its geometry. The first of these tasks, working with initial data and the constraint equations, is the focus of Chapter 8. The second task, involving evolution is the subject of Chapter 9. Chapter 7 discusses the ideas and methods which are used to carry out both of these tasks numerically, as well as some of the novel insights that have been obtained from this numerical work.

It is not surprising for the field equations of a physical theory to include constraint equations. Maxwell's equations, for example, include the constraints $\nabla \cdot B=0$ and $\nabla \cdot E=$ $4 \pi \rho$. However, the Einstein constraints (see Chapter 8) are much harder to handle than their Maxwellian counterparts, so considerable mathematical research has gone into working with them over the years.

The approach which has been most successfully used over the years for constructing and analyzing solutions of the constraint equations is the conformal method. Based on the work of Lichnerowicz [60] and York [61], the conformal method splits the initial data into "seed data", which can be freely chosen, and "determined data", which one obtains if possible by solving the constraint equations with the seed data specified (see Chapter 8 for details). The goal of the conformal method is two-fold: to obtain an effective parametrization of the "degrees of freedom" of the gravitational field, and to construct initial data sets which incorporate the physics of interest. 
For initial data with constant mean curvature (CMC), the conformal method works very well. This is true for data on compact manifolds, for asymptotically Euclidean (AE) data, and for asymptotically hyperbolic $(\mathrm{AH})$ data. It also works very well for data sets with sufficiently small mean curvature (near-CMC), so long as there are no conformal Killing fields present. However, more generally, much less is known. There are special classes of non-CMC seed data for which the conformal method has been shown to work [62], but there are others for which it appears to behave quite badly [63]. While to date these latter classes are quite restricted, the signs of trouble are clear: It may well be that the conformal method is effective for CMC and near-CMC initial data, but is ineffective more generally.

Are there modifications of the conformal method which might get around these looming difficulties? We first note that while the conformal thin sandwich method [64] is a popular alternative to the conformal method among numerical relativists, Maxwell has shown [65] that these two methods are mathematically equivalent - they succeed or fail together. Thus, one expects that more substantial modifications may be necessary to obtain a method which works for all solutions of the constraint equations. Maxwell [66] has proposed one possible direction for such modifications, in terms of his "drift" vector treatment of the mean curvature in the seed data, but much more work is needed to determine if this or any other modification is likely to be successful.

While the conformal method has to date been the dominant tool used for constructing and analyzing solutions of the constraint equations, there are other approaches available. Most notable are the gluing techniques, which are designed to combine two or more known solutions of the constraints into a single one. Roughly speaking, there are two types of gluing techniques which have proven to be effective. Connected sum gluing starts with a pair of smooth solutions of the constraints, chooses a point in each, and produces a new solution of the constraints on the connected sum manifold ${ }^{6}$ which is identical to the original solutions away from the chosen gluing points. This gluing technique [67, 68] works for any pair of initial data sets - compact, $\mathrm{AE}$, or $\mathrm{AH}$ - so long as a certain non degeneracy condition (see [68]) holds at the gluing points. It also works for most coupled-in matter source fields. It has been used to prove a number of interesting results, including that there exist maximally-extended globally hyperbolic solutions of the constraints containing no CMC Cauchy surfaces.

The other type of gluing was introduced in 2000 in the landmark work of Corvino [69], in which it is shown that for any time-symmetric initial data set which solves the constraints and is asymptotically Euclidean in a suitable sense, and for any bounded open interior region $W$, there is a new solution of the constraints which is identical to the original in $W$, and is identical to a subset of the Schwarzschild initial data outside of some bounded set containing $W$. The surprise here is that quite general interior gravitational configurations can be smoothly glued to a Schwarzschild exterior. Equally surprisingly, Corvino and Schoen show in [70] (see Chapter 8) that this result extends to non-time-symmetric initial data, with the exterior region being Kerr rather than Schwarzschild. Based on this work, one can show that there is a large class of asymptotically flat solutions of the Einstein equations which admit the conformal compactification (and the corresponding $\mathcal{I}$-structure) of the sort proposed by Penrose for studying asymptotically flat solutions.

\footnotetext{
${ }^{6}$ Roughly speaking, the connected sum of a pair of manifolds is obtained by removing a neighborhood of a point from each, and then adding a tube which connects the manifolds where the neighborhoods have been removed.
} 
Very recently, a further surprising variant of Corvino-Schoen type gluing has been developed by Carlotto and Schoen. They show [71] that, just as one can construct a solution of the constraints by gluing an arbitrary interior region across an annular transition region to an exterior Schwarzschild or Kerr region, one can also do so by gluing an arbitrary solid conical region (stretching out to spatial infinity) across a transition region to a region of Minkowski initial data with a conical region removed. Moreover, one can do this without significantly changing the ADM mass. Both the Carlotto-Schoen conical gluing and the Corvino-Schoen annular gluing [72], can be used to produce $\mathrm{N}$-body initial data sets of specific design which satisfy the constraints. The evolutions of such N-body initial data sets have not yet been studied.

In addition to the conformal method and the gluing techniques, there is one other procedure which has been used to produce solutions of the constraints. Designed to construct solutions with fixed interior boundaries, Bartnik's "quasispherical method" [73] chooses coordinates in such a way that one of the constraints takes the form of a parabolic (heat-type) equation with the radial coordinate as "time". Specifying data on the interior boundary, one "evolves" outward towards $r \rightarrow \infty$. Originally proposed as a tool for studying the Bartnik quasilocal mass, the quasispherical method has been used as well for constructing dynamical horizon initial data sets [74]. It would be useful to explore possible further applications.

The development of procedures for constructing solutions of the constraint equations is only one part of the broad scale of research focussed on initial data sets and the constraints. Another very important area aims to understand the energy, momentum and angular momentum of isolated gravitational systems, both for the system as a whole and for specified subregions. Such notions, well understood in Newtonian physics, are not obvious in general relativity, especially for non-isolated subregions. Globally, for a data set which is asymptotically Euclidean, the ADM formalism [75] provides definitions which are correct from the Hamiltonian perspective, and are theoretically useful. In particular, the global ADM energy $E_{A D M}$ and the global ADM momentum $P_{A D M}$ are both crucial for the statement and proof of two of the major results of mathematical relativity: the Positive Mass Theorem and the Penrose Inequality Theorem. As discussed in Chapter 8, the first of these states that for every $\mathrm{AE}$ initial data set satisfying the constraints, the corresponding $E_{A D M}$ and $P_{A D M}$ satisfy the inequality $E_{A D M} \geq\left|P_{A D M}\right|$, with equality holding if and only if the initial data set generates Minkowski spacetime. The Penrose Inequality Theorem, which thus far has been proven only for time-symmetric initial data sets, states that for time-symmetric AE solutions of the constraints the ADM mass $\mu_{A D M}:=\sqrt{E_{A D M}^{2}-\left|P_{A D M}\right|^{2}}$ satisfies the inequality $\mu_{A D M} \geq \sqrt{\frac{A}{16 \pi}}$, where $A$ is the surface area of the outermost horizon in the data set.

The proofs of both of these theorems - the first by Schoen and Yau [76] and then independently by Witten [77] during the late 1970s, the second by Huisken and Ilmanen [78] and then independently by Bray [79] at the turn of the millennium-were major triumphs of geometric analysis. To a large extent, they showed mathematicians working in geometric analysis that general relativity could be a fertile source of mathematically deep - and solvable - problems. Indeed, formulating and proving a version of the Penrose Inequality Conjecture for non-time-symmetric initial data is an outstanding open problem in mathematical relativity and geometric analysis.

While the definitions, major results, and major open questions regarding global quantities like $E_{A D M}$ are agreed upon by most relativists, much less is settled for the localized quantities. There is agreement regarding the properties that a "quasi-local mass" should 
have. Yet, as discussed in Chapter 8, there is a wide variety of possible definitions, including the Brown-York mass, the Liu-Yau mass, the Wang-Yau mass, the Hawking mass, and the Bartnik mass. In addition, there are definitions of quasi-local mass relying on isolated horizons and on dynamical horizons. All have their virtues and their difficulties, and their studies motivate a variety of challenging problems in geometric analysis.

One of the geometric structures which arose as a useful tool for the Schoen-Yau proof of the Positive Mass Theorem is the marginally outer trapped surface or MOTS. In a spacetime, an embedded two-dimensional spacelike surface $\Xi$ is a MOTS if one of the two families of future-directed null geodesics orthogonal to $\Xi$ has vanishing expansion everywhere along $\Xi$. Such behavior is closely tied to that of an apparent horizon, and consequently (through Hawking-Penrose-type singularity theorems) to the development of a black hole. One also notes that if $\Xi$ is contained in a time-symmetric initial data set, then it must be a minimal surface in that Riemannian manifold.

Given the strong tie between MOTS and black holes, and between MOTS and minimal surfaces, it is not surprising that the analysis of MOTS - conditions for their existence in a given spacetime, conditions for their stability, restrictions on their allowed geometry-has proven to be very rich. Chapter 8 covers these topics extensively, including an outline of a very nice MOTS-based simplification of the proof of the Positive Mass Theorem [80].

From a physical point of view, the analysis of initial data sets and solutions of the Einstein constraint equations is all about specifying physically interesting initial configurations of gravitational systems, understanding the properties of these systems, and identifying the degrees of freedom of the gravitational field if possible. Correspondingly, from a physical point of view the analysis of the evolution equations is all about determining how (according to general relativity) such initial configurations evolve in time: Do they form black holes? Do they form singularities? If they produce gravitational radiation, what forms might the radiation take for different initial configurations?

Choquet-Bruhat's well-posedness theorem shows that (in suitable coordinates) Einstein's field equations constitute a (nonlinear) hyperbolic PDE system. However, there are two very important ways in which the Einstein system differs from other geometrically-based hyperbolic PDE systems such as the Yang-Mills equations or wave maps on Minkowski spacetime. First, while the Yang-Mills and wave map examples have a fixed background spacetime which can be used to define long-time existence, singularities, and energy functionals unambiguously, with Einstein's theory the spacetime evolves along with the solution, so these concepts are much harder to pin down. Second, since general relativity uses solutions of Einstein's equations to model (classical) physical phenomena, there is physical interest in individual solutions and their evolution; for Yang-Mills and wave maps this is not the case.

These two distinguishing characteristics of Einstein's equations have had two important consequences. The lack of a fixed background has made it difficult until recently to use some of the standard ideas and tools of nonlinear hyperbolic PDE analysis. The physical interest in individual solutions has made the pursuit of numerical simulations of solutions a very important part of general relativity since the 1970s.

While there was little progress in either the numerical or the hyperbolic PDE analysis of Einstein's equations in the two decades following Choquet-Bruhat's theorem, important insights were obtained regarding singularities (see [81]). It was known from the study of explicit solutions that singularities (in the sense of unbounded curvature) can develop, but it was not known if this was just an artifact of the symmetries of solutions such as FLRW and Schwarzschild. The Hawking-Penrose "singularity theorems", which were proven using 
the properties of geodesic congruences in curved spacetimes, did show that if one identifies geodesic incompleteness with singularities, then singularities occur in large classes of solutions. However, in making this identification, one obscures the issue of the physical meaning of a singularity occurring in a given spacetime. For example, in Taub-NUT spacetimes, geodesic incompleteness marks the breakdown of causality (with closed timelike paths appearing), while in FLRW spacetimes, geodesic incompleteness occurs because of unbounded tidal curvature.

Restated as the Strong Cosmic Censorship (SCC) conjecture, this issue regarding the generic nature of solutions of Einstein's equations which are geodesically incomplete is one of the outstanding questions of mathematical relativity. Proposed during the late 1960s by Penrose, the SCC conjecture can be stated as follows: Among all sets of constraintsatisfying initial data whose maximal developments ${ }^{7}$ are geodesically incomplete, all but a set of measure zero have unbounded curvature, and cannot be extended ( across a "Cauchy horizon") as non-globally hyperbolic solutions. In fact there are many families of globally hyperbolic solutions which are geodesically incomplete, have bounded curvature, and can be extended across Cauchy horizons [84]; this is fully consistent with SCC, since its claim is that generic geodesically incomplete solutions have unbounded curvature and cannot be extended; SCC allows for some solutions to behave otherwise.

As discussed in Chapter 9, Strong Cosmic Censorship has been carefully formulated and proven for a number of restricted families of solutions, such as the $T^{3}$ Gowdy solutions [85]. Whether or not it holds generally is a open question.

Also open and also one of the central questions of mathematical relativity is the Weak Cosmic Censorship (WCC) conjecture. Despite its name, the WCC conjecture (also due to Penrose) neither implies nor is implied by SCC. It can be stated as follows: Among all sets of constraint-satisfying initial data sets whose maximal developments are asymptotically flat and contain a curvature singularity, in all but a set of measure zero there is an event horizon which shields the singular region from observation by asymptotic observers. In other words, WCC conjectures that in solutions of Einstein's equations, gravitational collapse generically leads to the formation of a black hole. As with SCC, genericity is a key part of the conjecture: There are gravitationally collapsing solutions with naked singularities, but WCC conjectures that this does not generically occur. Restricted families of solutions for which WCC has been proven are discussed in Chapter 9.

Once it has been determined that a nonlinear hyperbolic PDE system has solutions which evolve from regular initial data and become singular in finite time, one of the main questions becomes the stability of solutions. That is, fixing a particular solution $\Psi$, is it true that the evolution of every solution with initial data close to that of $\Psi$ must evolve essentially as does $\Psi$ ? In addition to its mathematical interest, stability is important physically, since $\Psi$ is useful in modeling physical systems only if it is stable in this sense.

For Einstein's equations, stability is not at all obvious. For example, considering Minkowski spacetime, does one expect very small gravitational perturbations of flat data to disperse (stability), or to concentrate and form a black hole (instability)?

\footnotetext{
${ }^{7}$ As proven by Choquet-Bruhat and Geroch [82], for every initial data set which satisfies the constraints, there is a unique (up to diffeomorphism) globally hyperbolic spacetime which is evolved (via Einstein's equations) from that initial data, and which extends all other globally hyperbolic solutions evolved from the same data. This spacetime is called the maximal development of that data set. A recent new proof 83] of this theorem avoids the use of Zorn's Lemma.
} 
The Christodoulou-Klainerman proof of the stability of Minkowski spacetime [86] is one of the iconic results in mathematical relativity. Besides resolving an important question concerning Einstein's theory, this proof showed that many of the difficulties impeding the analysis of Einstein's equations as a nonlinear PDE system could be overcome. It showed that coordinate freedom could be controlled using null foliations, and it showed that the Bel-Robinson tensor could be used to construct an effective energy functional for Einstein's theory (see details in Chapter 9). Many of the ideas developed in this work were used subsequently by Christodoulou [87] to prove that certain ("pulse") classes of regular initial data necessarily evolve into spacetimes with trapped surfaces and therefore very likely into black holes.

Minkowski spacetime is not the only solution of Einstein's equations which has been shown to be stable. Almost ten years before the Christodoulou-Klainerman work, Friedrich proved [88] that the de Sitter spacetime, which is a solution of the Einstein equations with positive cosmological constant, is stable. To do this, he relied on his conformal reformulation of the field equations (based on the higher-order Bianchi identities) and the conformal properties of the de Sitter spacetime, thereby avoiding some of the problems encountered in proving longtime existence for solutions near Minkowski spacetime. Stability has also been proven for a number of solutions which have a steady or accelerating rate of expansion to the future. As discussed in Chapter 9, Andersson and Moncrief [89] have shown that the Milne spacetimes ${ }^{8}$ are stable. In this work, a coordinate choice which results in an elliptic-hyperbolic form for the evolution equations plays a crucial role. In more recent work, Ringström [90, 91] has examined solutions of certain Einstein-scalar-field theories which are characterized by accelerated rates of expansion and shown that these solutions are stable. Followup work [92, 93] suggests that his techniques are robust for expanding solutions.

One of the most challenging open questions in general relativity is whether the Kerr solutions are stable. This is a mathematically very rich problem, which has motivated a significant amount of research (some discussed in Chapter 9). Since the Kerr solutions are believed by many to accurately model the final state resulting from the gravitational collapse of large mass stars, it is very important physically to determine if the Kerr solutions are stable. We note that general perturbations of Schwarzschild solutions are not expected to evolve to Schwarzschild solutions; rather they are expected generally to evolve to Kerr solutions.

Stability and cosmic censorship are not the only questions arising in the study of the longtime behavior of solutions of Einstein's equations. One would like to explore the long-time, asymptotic behavior of families of solutions which are not necessarily small perturbations of known solutions like Minkowski, Kerr, Milne, or FLRW. While much less is known about how to do this, two very active areas of research that are discussed in Chapter 9 could be very helpful. In part 2, efforts to reduce as much as possible the regularity (differentiability) needed for well-posedness are discussed. Such efforts, which have steadily progressed from Choquet-Bruhat's original theorem (requiring smooth data) to the recent remarkable $L^{2}$ bounded curvature version [94], are important for long-time evolution studies because they establish crucial break-down and continuation criteria for evolving solutions. That is, if one

\footnotetext{
8 The Milne spacetimes are obtained by topologically compactifying the constant mean curvature hyperboloids in the future lightcone of a point in Minkowski spacetime. Together these CMC hypersurfaces foliate the spacetime, which is flat but is geodesically incomplete to the past. To the future, the CMC hypersurfaces have a steady rate of expansion.
} 
can prove well-posedness for data with bounded curvature, then one knows that evolution continues so long as indeed the curvature remains bounded. Combining results of this sort with the control of certain energy functionals (designed to control curvature) would lead to long-time existence.

A scenario for defining and establishing control of energy functionals is outlined in part 3 of Chapter 9. For solutions involving negative scalar curvature metrics, one can choose certain gauges and coordinates, and carry out a reduction ${ }^{9}$ which results in a Hamiltonian formulation with a well-defined, monotonic Hamiltonian (energy) functional. Combining this reduction with lessons learned in studying long-time existence for the Yang-Mills equations [95] could lead to very interesting insights into the dynamics of Einstein's equations.

In mathematical analyses of PDE systems, the emphasis is usually on understanding the properties and the behavior of large sets of solutions. For a PDE system used to model physics, one is interested in individual solutions as well. Numerical simulation of solutions is crucial for such studies; consequently much effort has gone into developing numerical relativity since the early 1970 s.

Although the techniques used for carrying out numerical simulations are very different from those used in performing mathematical analysis, the same basic issues - controlling diffeomorphisms, choosing gauges and coordinates, distinguishing physical effects from coordinate effects, dealing with a non-fixed background spacetime-have caused difficulties in both enterprises. Consequently, advances in one of these enterprises have often been very helpful in the other. A key example of this is the mathematical development of generalized harmonic coordinates as a tool for proving well-posedness, followed by the crucial role played by this coordinate choice in carrying out the first stable numerical simulation of a binary black hole coalescence [96], in turn followed by the use of generalized harmonic coordinates in proving the stability of solutions with accelerated expansion [90].

Throughout most of its history, from the 1960s to the present, numerical relativity has been primarily focused on simulating the coalescence of compact binaries, and determining the consequent generation of gravitational radiation as well as the properties of the remaining object. This is largely because of the central role such collisions are expected to play in generating radiation observable by LIGO, Virgo and other detectors, and the need to understand the detailed features of this radiation if it is to be detected in practice. Despite significant investments in both human effort and resources, it was a long trail from the earliest efforts to simulate black hole collisions by Hahn and Lindquist in 1964 [97] and the early development work of Smarr and Eppley during the 1970s, to the first stable simulations of these collisions by Pretorius [96] and independently by groups led by Campanelli [98] and by Centrella [99] in 2005, culminating in the current systematic production of simulations of a large parameter space of collisions of binary systems, including neutron stars as well as black holes. Chapter 7 presents much of the story of this difficult (and adventurous) journey.

In addition to its role in simulating sources of gravitational radiation, numerical relativity has been very useful for other explorations of general relativity. Perhaps most noteworthy is the discovery in such simulations by Choptuik [100] of critical phenomena in gravitational collapse. Critical phenomena are found by considering the evolutions of each of a oneparameter $(\lambda)$ family of initial data sets, with those solutions evolving from data with

\footnotetext{
${ }^{9}$ Such a reduction effectively solves some of the constraints and implements the coordinate and gauge conditions in such a way that the evolution projects down to a phase space with fewer dynamical variables. See part 3 of Chapter 9 for details.
} 
large $\lambda$ collapsing to form black holes, and those solutions evolving from data with small $\lambda$ dispersing. At the transition value $\lambda_{c}$ between data leading to black holes and data leading to dispersion, the evolved solution has been found to have special features including certain types of (continuous or discrete) time symmetry described by scaling laws. Critical behavior has been observed (via simulations) to occur for essentially all such choices of one-parameter families of data ("universality") and has been found to occur for Einstein's equations coupled to a very wide variety of source fields. Details are presented in Chapter 7. Interestingly, while the evidence for critical behavior from numerical simulations is overwhelming, there has been no mathematical proof of its existence.

Also noteworthy is the significant role which has been played by numerical simulations in the study of the behavior of the gravitational field near the singularity in families of solutions defined by $T^{2}$ or $U(1)$ spatial isometry (including the Gowdy solutions). These simulations, initiated by Berger and Moncrief [101], indicated the prevalence of "AVTD" behavior in Gowdy solutions as well as in polarized $T^{2}$ symmetric and polarized $U(1)$ symmetric solutions. That is, as one evolves toward the singularity in these solutions (at least in certain chosen coordinate systems), spatial derivative terms in the evolution equations appear to be dominated by time derivative terms; consequently, an observer following a coordinate path sees the fields evolve more and more like a Kasner solution, with each observer seeing an independent Kasner. This somewhat peculiar behavior was first described during the 1960s by Lifshchitz and Khalatnikov who predicted that it should characterize large classes of solutions. In later years with Belinskii [102] they amended their prediction to claim that solutions with singularities would generically exhibit what is now known as "BKL" or "Mixmaster" behavior: each observer would see an endless sequence of Kasnerlike evolutions, punctuated by "bounces" leading from one Kasner era to another. While it was widely believed during the 1980s that neither AVTD nor BKL behavior was likely to be seen, the numerical work by Berger, Moncrief, Garfinkle, Weaver, Isenberg, and others during the 1990s has supported the contention that AVTD and BKL behavior are there, but with the added feature of "spikes". Indeed, it has now been proven by Ringström [103] that $T^{3}$ Gowdy solutions are generically AVTD; this demonstration is a crucial step in his proof that Strong Cosmic Censorship holds for these Gowdy solutions.

Chapter 7 discusses a number of other important numerical relativity projects which have indicated surprising physical phenomena in general relativity. Most notable is the apparent instability of anti-de Sitter spacetime which has been discovered numerically [104]. This result is very surprising for two reasons: i) it contrasts with the proven stability of Minkowski spacetime and De Sitter spacetime, and ii) it has been shown that anti-DeSitter spacetime is linearly stable. ${ }^{10}$ Also very interesting are the studies of the complex dynamics of perturbations of black strings in $4+1$ dimensions [105]. In both of these cases, mathematical questions have motivated the studies, and the numerical work has provided very strong indications of unambiguous answers to these questions.

One area not discussed in these Chapters in which the partnership of numerical and mathematical work has been very useful is in the study of the strong field regime during the formation and coalescence of black holes. Mathematically, the definition of black holes has traditionally been associated with event horizons, the future boundaries of the causal past of future null infinity. They separate the spacetime region from which light can escape to

${ }^{10}$ A solution is linearly stable if the corresponding linearized Einstein equation operator has negative eigenvalues. 
infinity from those regions from which light can never do so. Unfortunately, an immediate consequence is that the notion of an event horizon is not only extremely global but it is also teleological; one has to know the entire spacetime before one can locate it. In particular, an event horizon can grow in regions which have weak - or even zero-spacetime curvature, in anticipation of a future gravitational collapse. Therefore in the study of gravitational collapse or dynamics of compact objects, one cannot locate event horizons during evolution. In practice, numerical simulations have used "apparent horizons" on the chosen family of Cauchy hypersurfaces to indicate incipient black holes because neither of the two light fronts emanating from such surfaces is expanding; light is trapped at least instantaneously. However, this notion is tied to the choice of Cauchy hypersurfaces. To get around this issue, one can consider spacetime world tubes of apparent horizons, known as dynamical horizons if they evolve, and isolated horizons if they do not [106 109]. As black hole markers, these structures have the advantage that they are determined by the local spacetime geometry in their immediate vicinity; they do not require knowledge of the full spacetime and in particular they do not exist in a flat spacetime. Dynamical horizons and isolated horizons have been studied both analytically and numerically. From these studies, tools have been developed to extract physical information, such as the mass, angular momentum, and (source) multipole moments of black holes, even in dynamical regions, in a coordinate invariant fashion. These structures have also made it possible to meaningfully compare results of simulations that use different initial data, coordinates and foliations in the strong field region. Finally, they provide analytical tools to probe how back holes approach their final, universal equilibrium state, represented by the geometry of the Kerr horizon, and if the process has universal features [110, 111]. While these developments were inspired by simulations, they have also had interesting applications on the analytical side, including significant generalizations of the laws of black hole mechanics [112, 113] to fully dynamical situations [108], black hole evaporation [114] and calculations of horizon entropy using quantum geometry, as summarized in Chapter 11.

One very elusive issue for which dynamical horizons could be helpful is to find a comprehensive definition of black holes which makes sense in dynamical spacetimes, as well as in spacetimes which are not asymptotically flat [115]. One problem with using dynamical horizons, however, is that they are generally not unique [116]; consequently they are not good candidates for canonically locating the surface of a black hole.

Finally, we note one other very elusive physical issue which calls for both numerical and mathematical studies, in partnership, concerning gravitational waves. As explained in Part II, although Einstein analyzed gravitational waves in the linearized approximation and derived the quadrupole formula soon after his discovery of the field equations, there was considerable confusion over the next three decades regarding the physical reality of gravitational waves because it was difficult to disentangle physical effects from coordinate artifacts. The situation was clarified only in the sixties when the notion of Bondi news was shown to provide a coordinate invariant characterization of gravitational radiation [117]. That framework continues to provide the conceptual foundations of the gravitational wave theory discussed in Chapter 6. However, it assumes a zero cosmological constant; $\Lambda=0$. This is a problem, because cosmological observations have established that $\Lambda>0$ is to be preferred and, surprisingly, we do not yet have a satisfactory analog of the Bondi news, or viable expressions of energy, momentum and angular momentum carried by gravitational waves in full, non-linear general relativity for $\Lambda>0$. Indeed, even the asymptotic symmetries that are needed to introduce these notions do not extend to the $\Lambda>0$ case. In cosmological 
applications, it has been sufficient just to use linearized gravitational waves. Similarly, for isolated systems such as compact binaries or collapsing stars, working with $\Lambda=0$ should be an excellent approximation in most calculations since the observed value of $\Lambda$ is so small. However, as of now, we do not have any theory for $\Lambda>0$, whose predictions are to be approximated by the $\Lambda=0$ calculations. Only when we have this theory and a detailed understanding of how $\Lambda$ affects physical quantities can we be confident that the current $\Lambda=0$ calculations do approximate reality sufficiently well. Moreover, there may well be subtle effects - similar to the Christodoulou memory effect in the $\Lambda=0$ case - that have eluded us and which will be uncovered only when the $\Lambda>0$ case is well understood.

For a number of years following its pristine birth one hundred years ago, general relativity was all too often admired and adulated rather than tested and put to work. Just as this has now changed in the experimental and observational realm, as reported in Parts I and II, we see here in Part III that this has dramatically changed in the mathematical and numerical realm as well. Einstein's theory is now recognized as a fresh source of interesting and challenging mathematical problems, and the successful solution of some of these problems has attracted increasing numbers of mathematicians with innovative skills. In turn, with more powerful analytical tools available, relativists have been able to explore the physical implications of general relativity more effectively. The intriguing mix of beautiful mathematics and profound physics, always an enticing feature of general relativity, is now a central reason for expecting rapid progress both in its use as an effective tool for studying gravitational physics, and its role as a challenging field of geometric analysis.

\section{PART IV: BEYOND EINSTEIN}

The remarkable advances summarized in the first three parts of this volume refer almost entirely to the well-established realm of classical general relativity (GR). However, Einstein [118] was quite aware of the limitations of his theory. In the context of cosmology he wrote, as early as in 1945,

"One may not assume the validity of field equations at very high density of field and matter and one may not conclude that the beginning of the expansion should be a singularity in the mathematical sense."

By now, we know that classical physics cannot always be trusted even in the astronomical world because quantum phenomena are not limited just to tiny, microscopic systems. For example, neutron stars owe their very existence to a quintessentially quantum effect: the Fermi degeneracy pressure. At the nuclear density of $\sim 10^{15} \mathrm{~g} / \mathrm{cm}^{3}$ encountered in neutron stars, this pressure becomes strong enough to counterbalance the mighty gravitational pull and halt the collapse. The Planck density is some eighty orders of magnitude higher! Astonishing as the reach of GR is, it cannot be stretched into the Planck regime; here one needs a grander theory that unifies the principles underlying both general relativity and quantum physics.

\section{Early developments}

Serious attempts at constructing such a theory date back to the 1930s with papers on the quantization of the linearized gravitational field by Rosenfeld [119] and Bronstein [120]. Bronstein's papers are particularly prescient in that he gave a formulation in terms of the 
electric and magnetic parts of the Weyl tensor and his equations have been periodically rediscovered all the way to 2002 [121]! Analysis of interactions between gravitons began only in the 1960s when Feynman extended his calculational tools from QED to general relativity [122]. Soon after, DeWitt completed this analysis by systematically formulating the Feynman rules for calculating the scattering amplitudes among gravitons and between gravitons and matter quanta. He showed that the theory is unitary order by order in perturbation theory (for summary, see, e.g., [123]). In 1974, 't Hooft and Veltman [124] used elegant symmetry arguments to show that pure general relativity is renormalizable to 1 loop but they also found that this feature is destroyed when gravity is coupled to even a single scalar field. For pure gravity, there was a potential divergence at two loops because of a counter term that is cubic in the Riemann tensor. However there was no general argument to say that its coefficient is necessarily non-zero. A heroic calculation by Goroff and Sagnotti [125] settled this issue by showing that the coefficient is $\left(209 / 2880(4 \pi)^{2}\right)$ ! Thus in perturbation theory off Minkowski space, pure gravity fails to be renormalizable at 2 loops, and when coupled to a scalar field, already at 1-loop.

The question then arose whether one should modify Einstein gravity at short distances and/or add astutely chosen matter which would improve its ultra-violet behavior. The first avenue led to higher derivative theories. Stelle, Tomboulis and others showed that such a theory can be not only renormalizable but asymptotically free [126]. But it soon turned out that the theory fails to be unitary and its Hamiltonian is unbounded below. The discovery of supersymmetry, discussed in Chapter 12, suggested another avenue: with a suitable combination of fermions and bosons, perturbative infinities in the bosonic sector could be canceled by those in the fermionic sector, improving the ultraviolet behavior. This hope was shown to be realized to 2 loops by Deser et al and Grisaru et al [127]. However, by the late 1980s a consensus emerged that all supergravity theories would diverge by 3 loops and are therefore not viable (see, e.g., [128]).

A series of parallel developments was sparked in the canonical approach by Dirac's analysis of constrained Hamiltonian systems. In the 1960s, this framework was applied to general relativity by Dirac, Bergmann, Arnowitt, Deser, Misner and others [130 134]. The basic canonical variable was the 3-metric on a spatial slice and, as discussed in Chapters 8-10, general relativity could be interpreted as a dynamical theory of 3-geometries. Wheeler therefore baptized it geometrodynamics [135, [136]. Wheeler also launched an ambitious program in which the internal quantum numbers of elementary particles were to arise from non-trivial, microscopic topological configurations and particle physics was to be recast as 'chemistry of geometry'. This led to interesting discoveries at the interface of topology and general relativity but the approach did not have notable success with the particle physics phenomenology.

A distinguishing feature of the canonical approach is that in contrast to perturbative treatments it does not split the metric into a kinematic background and a dynamical fluctuation. As a result, a number of conceptual problems were brought to the forefront which revealed the deep structural differences between general relativity and more familiar field theories in Minkowski spacetime. By now there is a near universal appreciation of the importance of background independence and of the necessity of facing the ensuing complications. However, this very feature made it difficult to use the standard techniques from QED to face the mathematical difficulties associated with the infinite number of degrees of freedom of the gravitational field. Consequently, most of the work in full 
quantum geometrodynamics remained rather formal. Detailed calculations could be carried out in the context of quantum cosmology where one freezes all but a finite number of degrees of freedom. Initially there was hope that quantum effects would tame the cosmological singularities of general relativity. However, this hope did not materialize; even in the simplest models the big bang could not be softened without additional 'external' inputs into the theory. The program also faced a sociological limitation in that the ideas that had been so successful in QED played no role: in a non-perturbative, background independent approach, it is hard to see gravitons, calculate scattering matrices and use virtual processes to obtain radiative corrections. To use a well-known phrase [137], the emphasis on geometry in the canonical program "drove a wedge between general relativity and the theory of elementary particles." Therefore, after an initial burst of activity, the quantum geometrodynamics program became rather stagnant.

A third avenue was opened in the mid 1950s: explorations of the effects of a classical gravitational field on quantum matter fields. Early work by Parker explored quantum fields in FLRW spacetimes [129]. As recent successes of inflationary scenarios discussed in Chapters 4 and 11 show, this choice was prescient. Indeed, this is the arena where we are most likely to first see the interface of gravity and quantum physics observationally. But this general area did not draw much attention until Hawking's seminal discovery in 1974 that quantum field theory (QFT) on a black hole background predicts that black holes emit quantum radiation and resemble black bodies when seen from infinity. Not only did the entire area of QFT in curved spacetime experience an explosion of activity but this discovery has served as a focal point for a great deal of research in all areas of quantum gravity over the last four decades.

\section{Current status}

Ideas developed in QFT in curved spacetimes have had a number of fascinating applications, ranging from the study of diverse aspects of the Casimir effect [138] to the feasibility studies of creating time machines by exploiting the violations of local energy conditions that are allowed in QFT [139]. Advances on the more fundamental side are discussed in Chapter 10. In general curved spacetimes, we do not have the Poincaré group to decompose fields into positive and negative frequency parts and select a canonical vacuum. Since there is no natural choice of a (Fock) representation of the canonical commutation relations, one is led to the more general setting of algebraic QFT. As a consequence, recent advances in QFT in curved spacetimes have brought to the forefront the essential conceptual ingredients of QFT that are often masked by the extraneous structure that happens to be available in Minkowski spacetime. As emphasized in Chapter 10, these developments have also shown that, beyond the special context of static spacetimes, techniques from Euclidean QFT cannot be carried over to calculate quantities of direct physical interest in the Lorentzian theory. This is an important message also for approaches to full quantum gravity although, unfortunately, it is often overlooked. On the physical side, quantum effects on curved spacetimes lead to unforeseen phenomena such as evaporation of black holes and emergence of the large scale structure of the universe from pure quantum fluctuations in the very early universe. Finally, advances in describing interactions have now elevated QFT in curved spacetimes to the same mathematical level as that enjoyed by rigorous QFT in Minkowski space. 
In quantum gravity proper, while both the perturbative and the canonical approaches reached an impasse by the early 1980s, they provided seeds for most of the subsequent developments. Although GR is perturbatively non-renormalizable, an effective field theory was developed systematically [140] and has had remarkable successes in the low energy regime, e.g., in the treatment of dynamics of compact binaries in classical GR [141] and in the computation of the leading corrections to the Newtonian potential. Therefore the problem of finding a viable quantum gravity theory can be rephrased as that of obtaining an appropriate completion of this theory in which the outstanding conceptual issues-such as the fate of the classical singularities of GR, the statistical mechanical accounting of black hole entropy, and the final stages of the black hole evaporation - can be analyzed systematically.

This quest has been undertaken in a number of directions. Each program adopts a different point of departure, treating certain aspects of the problem as more fundamental, and hoping that the remaining aspects can be handled successfully once there is a resolution of the key difficulties. Chapter 11 focuses on approaches which emphasize the dynamical nature of spacetime geometry and non-perturbative methods. The point of departure is GR. However, the fundamental degrees of freedom and the short-scale dynamics in the final quantum theory are quite different from those of GR and classical spacetimes and gravitons emerge only in a suitable limit.

The first of these programs is Asymptotic Safety, whose goal is to provide a specific ultraviolet completion of the effective field theory using Weinberg's generalized notion of renormalization. On the analytical side, non-trivial fixed points of the renormalization group flow have been obtained in 4 spacetime dimensions, even after allowing for 9 different gravitational couplings in addition to the coupling of gravity to matter fields. These results strongly suggest that much of the intuition derived from Goroff and Sagnotti's early results are tied to perturbation theory around Minkowski space. Consistent ultraviolet completions of the standard effective theory may well exist without having to invoke supersymmetry, higher dimensions or extended objects. Similarly, on the computational side it was widely believed that the renormalization group flows would inevitably lead to a 'crumpled phase' in which macroscopic spacetimes such as the one around us will not emerge in the infrared. Recent progress in Causal Dynamical Triangulations has shown that the 'crumpled phases' are not inevitable; theory does allow smooth, macroscopic spacetimes with small quantum fluctuations.

The second program discussed in Chapter 11 is Loop Quantum Gravity (LQG) which grew out of the canonical approach. Consequently, manifest background independence is at the forefront and the theory is again nonperturbative. However, emphasis is shifted from metrics to connections; the 'wedge' between general relativity and gauge theories governing other fundamental forces is removed. Indeed, the basic notions are taken directly from the Yang-Mills theory but without reference to a background spacetime metric. Rather surprisingly, this strategy leads to a unique kinematic setup in which one can overcome the mathematical obstacles faced in geometrodynamics and handle the infinite number of degrees of freedom rigorously. The Hilbert space is spanned by spin networks. Consequently, the fundamental excitations of geometry/ gravity are not gravitons which represent quantum fluctuations on a given background geometry. Rather, they are polymer-like threads that can be woven to create the geometry itself. Geometry is quantum mechanical in the most direct sense: geometric operators have discrete eigenvalues. This discreteness has a deep influence on dynamics. In covariant LQG - called the Spinfoam framework - it 
offers relief from ultraviolet infinities by banishing degrees of freedom at scales shorter than the Planck length. A priori, there could be infrared infinities but, surprisingly, if there is a positive cosmological constant, they can be removed by a natural quantum deformation of the local Lorentz group. LQG has had notable success both in the cosmological and black hole sectors of GR. In both cases, the underlying quantum geometry plays a key role. In particular, in the cosmological sector it naturally tames all strong curvature singularities even when matter satisfies all the energy conditions. Furthermore, the framework has extended the reach of observational cosmology all the way to the Planck regime through the development of QFT on quantum cosmological spacetimes. A similar extension is now being used to study Hawking radiation on quantum black hole spacetimes.

Chapter 12 summarizes advances based on supersymmetry, extended objects, higher dimensions and holography. Chronologically, at first the emphasis in the framework was on perturbation theory in Minkowski spacetime. The viewpoint was that the ultraviolet divergences of quantum gravity were signals that point particles and local quantum fields are over-idealized notions that become untenable at very high energies. Fundamental objects are strings, and interactions between them are just the simple processes of joining and splitting which naturally avoid the ultraviolet infinities of local QFT. Particles are merely excitations of strings. This immediately leads to an infinite tower of particles and fields but most of these excitations are so massive that they become relevant only at extremely high energies. Theoretical consistency implies that spacetime has to be 26-dimensional without supersymmetry and 10-dimensional with supersymmetry. At first it was believed that the extra dimensions could be compact and microscopic, and that there would be severe constraints on permissible compactifications as well as on the matter content and allowed interactions to make the theory unique. Therefore it was often heralded to be the "theory of everything'.

Further research showed that these expectations were overly optimistic, but at the same time it revealed richer structures: The theory turned out not to be unique but various consistent theories are related by certain dualities. It is now believed that there is probably a single theory and the known consistent theories represent its 'corners'. However, as of now its structure remains opaque; indeed, one does not even know what the fundamental principles behind it should be. This is reflected in its very name, 'M-theory', where $\mathrm{M}$ is often said to stand for 'Mystery'. Also, most of the work to date has been carried out in the framework of first quantization and the possible role of a second quantized string field theory remains unclear. But it is clear that the theory contains not only strings but also higher dimensional, extended, non-perturbative configurations, called $p$ branes. This enlargement of the theory opened a gate to incorporate extremal (and certain near-extremal) black holes in the string paradigm. Because of the 'non-renormalization' theorems, it is possible to calculate the number of certain string states at low energy and then correctly deduce the number of microstates of these black holes.

These considerations in turn suggested the possibility of a holographic picture in which string theory on asymptotically anti-de Sitter backgrounds could be regarded as being dual to certain conformal field theories on the boundary of these spacetimes. As discussed in Chapter 12, this AdS/CFT conjecture has led to a wealth of insights on the unity of the mathematical structures that underlie completely distinct physical systems such as quark-gluon plasmas and condensed matter systems. In addition, throughout its evolution, string theory has had unforeseen applications to diverse areas of mathematics. Given that 
these ideas extend the reach of gravitational physics to completely new territories in ways that were not even imagined before, it is fitting that the volume concludes with this Chapter.

Finally, it is important to emphasize that the content of Part IV does not do full justice to the field because space limitation did not allow us to include several promising advances. First, even in the areas covered in Chapters 10-12, in the spirit of this volume, authors focused on a few topics on which most significant advances have occurred over the last three decades or so. The second and more important omission is that several approaches to quantum gravity had to be left out entirely. In the first three Parts of this volume, which are based on well-established ideas and careful observations, the choice of what to include was easier to make. In the case of quantum gravity, the subjective element is much more pronounced simply because one is now forced to leave the safety net of ideas that are firmly grounded in GR. Promising directions that were left out include: i) Asymptotic quantization, which brought out the interplay between the Bondi-Metzner-Sachs group and infrared issues in full quantum gravity [142]; ii) Twisor theory based programs for calculating scattering amplitudes from past to future null infinity, that are now drawing a great deal of attention [143]. Twistor theory itself has provided a powerful bridge between the theory of partial differential equations and algebraic geometry which extends to (self-dual) Einstein's equations through Penrose's non-linear graviton construction [144]; iii) The Regge calculus approach, which parallels lattice QCD, but uses dynamical simplicial decompositions rather than lattices defined in a background geometry [145]; iv) Hořava-Lifshitz gravity, which sacrifices manifest local Lorentz invariance to achieve better ultraviolet behavior, hoping to recover it in the infrared limit [146]; v) Causal sets, in which one postulates that at a fundamental level one only has a discrete set of points with causal relations between them [147]; and vi) The Vassiliev higher spin theories, in which an infinite tower of massless higher spin fields are incorporated in a consistent manner [148]. One or more of these ideas may well lead to an ultraviolet completion of GR with desired features.

In this regard, it is instructive to look back at the events that celebrated the centennial of Einstein's birth some 35 years ago. The Princeton conference at the Institute of Advanced Study had two talks on quantum gravity; both on supergravity [149]. The Cambridge University Press volume [150] also had two Chapters, one that introduced the asymptotic safety program and the other that put all its emphasis on Euclidean quantum gravity. A year later, in his Lucasian Chair inaugural address, Hawking [151] suggested that the end of theoretical physics was in sight because $N=8$ supergravity was likely to be the final theory. The field has evolved rather differently! Fascinating as the advances over the last three decades have been, the reader would do well to keep this historic perspective in mind.

\section{Elephants in the room}

There is no question that today we understand the interface of gravity with quantum physics much better than we did in the mid 1980s when the approaches discussed in Chapters 11 and 12 first rose to prominence. Several unworkable ideas have been weeded out and, as our summary indicates, concrete advances have provided us with novel insights. It is therefore fitting that the review articles and status reports tend to be upbeat, exuding confidence. But it is also clear that the end of quantum gravity is not yet in sight. We, the Editors, would be remiss if we do not venture to say why. 
The leading approaches use diverse points of departure and have strikingly different perspectives as to what is most fundamental and what can be revisited later. The mathematical techniques they use also vary significantly. Given the difficulty of the task, this diversity is of course both healthy and essential. As our summary of early developments shows, this diversity has been a hallmark of this field for over 50 years. Today, the practitioners are even more passionate about the choices their approach makes. However, as a result, whereas other areas of gravitational science have witnessed a convergence of ideas and coming together of previously distinct communities, in quantum gravity the communities have drifted further apart. As active communications become less frequent, slowly but steadily the tendency to ignore the elephants in one's room increases. At the same time, it seems more and more natural to think of other viewpoints as untenable. To make this point more concrete, we will provide a few illustrative examples from the main programs discussed in this volume.

In the approaches based on unification, ideas that lead to mathematically rich structures play a dominant role. Consequently, as we discussed above, these approaches have led to unforeseen insights into the mathematical unity in the description of diverse physical systems. However, this success also seems to have fueled a tendency to ignore the issue of whether the central ideas behind these approaches are realized in our physical universe. In particular, there is little hesitation in building a quantum theory of gravity by demanding that it have supersymmetry, higher dimensions, infinite towers of particles and fields and a negative cosmological constant at its very foundation. To researchers outside quantum gravity the strategy seems surprisingly indifferent to the current state of observations, rather akin to searching for the key under a lamppost irrespective of where it was actually lost. It is true that some of these ideas are motivated by the Kaluza-Klein theory where extra dimensions are meant to be microscopic and curled up, remaining invisible until we reach energies near the Planck scale. But in detailed explorations this primary restriction is often set aside. For example, it is rarely imposed while studying higher dimensional solutions in these frameworks. More importantly, in the most commonly used versions of the AdS/CFT conjecture, symmetry requirements force the extra dimensions to be very large; the radius of the compactified internal spheres is the cosmological radius! If we lived in such a universe, the internal dimensions should be as readily observable as the 'normal' 4 spacetime dimensions. Taken together, these assumptions push one to a paradigm whose relevance to the physical issues of quantum gravity in the actual universe we inhabit becomes increasingly obscure.

Approaches developed primarily by researchers from the GR community make a serious attempt to base their foundations only on the well established principles of GR and QFT. They tend to take the gravity/geometry duality seriously and aim to understand the quantum nature of geometry first, postponing the issue of coupling to matter to a second stage. This strategy has had notable success in the asymptotic safety program. However, in this approach exploration of the quintessentially quantum gravity issues - such as the origin of black hole entropy and the fate of the most vexing singularities of GR - is still at a preliminary stage. Furthermore, whereas at a fundamental level the program refers to renormalization group flows in the infinite dimensional space of permissible theories, in practice it seems unlikely that one would be able to go beyond finite truncations in any foreseeable future. Therefore the question of whether the program truly provides the promised ultraviolet completion is likely to remain open for a long time.

LQG has also advanced by making truncations. However, as in the concrete calculations of QED or QCD, truncations refer to physical problems of interest, such as the Planck scale physics of the very early universe or quantum properties of black holes. In these 
truncated sectors, the theory has had notable success. But, as discussed in Chapter 11, the issue of dynamics in full LQG is still far from being settled. Spinfoams provide a natural framework to explore it and certain Spinfoam models have had notable success. But a number of fundamental issues remain: Does the natural expansion used to calculate 'transition amplitudes' converge? Is a continuum limit needed and, if so, how exactly is one to take it? Do the proposals to incorporate matter in Spinfoams work in detail? An equally important open issue is to make direct contact with low energy physics. Since one begins with quantum geometry in the Planck regime and then descends to the low energy world, this problem is highly non-trivial. There are numerous preliminary, encouraging results, such as the calculation of the graviton propagator starting from a fully non-perturbative and background independent setting. But the relation to the effective field theory beyond the leading approximation remains unclear. Until there is a solid bridge linking non-perturbative dynamics to the well-developed effective theory in detail, physical viability of the approach will remain uncertain.

\section{Epilogue}

While writing a review article on special relativity in 1907, Einstein realized that Newtonian gravity is incompatible with special relativity and set himself the task of resolving this conflict by creating a grander synthesis from which the two theories would emerge as limiting cases. Just eight years later, he arrived at the finished solution and for the last century, physical scientists from a broad array of disciplines have been happily engaged in investigating its content! Work on unifying GR with quantum physics, on the other hand, has seen many twists and turns; periods of euphoria followed by despair at conceptual impasses, or Nature's stubborn refusal to use structures that seem compelling to the practitioners. Einstein's spectacular success is in striking contrast with the time and effort that has been devoted to this endeavor.

But it is important to note that progress in physical theories has more often mimicked the development of quantum theory rather than general relativity. More than a century has passed since Planck's discovery that launched the quantum. Yet, the theory is incomplete. We do not have a satisfactory grasp of the foundational issues, often called the 'measurement problem'. Nor do we have a single example of a mathematically complete, interacting QFT in 4 dimensional Minkowski spacetime. A far cry from general relativity that Einstein offered us in 1915! Yet, no one would deny that quantum theory has been extremely successful; indeed, its scientific reach vastly exceeds that of general relativity.

Thus, while it is tempting to wait for another masterly stroke like Einstein's to deliver us a finished quantum gravity theory, it would be more fruitful to draw lessons from quantum physics. There, progress occurred by focusing not on the 'final' theory that solves all problems in one fell swoop, but on concrete physical problems where quantum effects were important. Experience to date indicates that the same will continue to be true for quantum gravity in the foreseeable future.

What could hasten progress along this path? So far individual programs have been driven by internal criteria. More significant advances could occur by critically examining common elements they share as well as tensions between the ideas that lie at their foundations.

For example, although these programs start with very different viewpoints and assumptions, they feature a curious dimensional reduction at the Planck length [152]. In LQG, the fundamental excitations of quantum geometry have 1 spatial dimension whence Spinfoams 
are 2-complexes. In Asymptotic Safety one has a running spectral (spacetime) dimension which (equals 4 in the infrared but) approaches 2 in the ultraviolet. In perturbative string theory, point particles are replaced by 1 (spatial) dimensional strings, propagating on a classical spacetime, say, Minkowski space. In the linearized approximation off Minkowski space, the LQG excitations have the same massless particle content as in bosonic string theory - a dilaton, an antisymmetric tensor and a spin-2 excitation - to begin with, and the graviton is extracted by imposing linearized Einstein constraints. Is there perhaps a deep reason why qualitatively similar 2-dimensional structures arise even when the starting points are so different?

The next set of issues is related to ultraviolet finiteness. Since the late 1980s there has been a strong belief in the string theory community that local interactions between point particles à la QFT would inevitably lead to ultraviolet divergences and the theory is rendered perturbatively finite by using strings instead. There has been considerable research on this issue especially in recent years. However, as the number of loops grow, there is an increasing number of ambiguities (because of the super-moduli measure needed) in the calculation and therefore some experts continue to believe that the issue of order by order perturbative finiteness is still open in string theory [153]. On another front, recent work on supergravity, described in Chapter 12 , has shown that the $\mathrm{N}=8$ supergravity in 4 spacetime dimensions is finite to 4 loops, contrary to the near unanimous expectations in the 1980s [128]. Some experts in supergravity have suggested that there could well be symmetries that have remained hidden so far that could make supergravity finite to all orders. Support for the general idea of hidden symmetries comes also from Vassiliev's higher spin theories. Overall, there is a small but growing community that believes that what is fundamental for finiteness is a sufficiently large and subtle symmetry group rather than extended objects. Thus, there is a healthy tension concerning the issue of what drives finiteness even at the level of perturbation theory.

The tension continues beyond perturbations. In LQG, background independence leads to a rather sophisticated quantum geometry with the property that there are no degrees of freedom below the Planck length, ensuring ultraviolet finiteness. In string theory, the sum in the perturbation expansion diverges [154] requiring a non-perturbative treatment. As we saw, a highly successful candidate is available in the sector of the theory with a negative cosmological constant: the AdS/CFT conjecture, where string theory in the bulk is equivalent to a finite field theory on the boundary. ${ }^{11}$ Note that this AdS/CFT correspondence also provides a background independent definition of string theory in the asymptotically AdS sector. But whereas the mechanism taming the ultraviolet regime is provided by a specific quantum Riemannian geometry in LQG, in string theory it is provided by holography. Is there nonetheless a deep connection between them? If so, it may be helpful in, e.g., the analysis of the physical cosmological singularities in string theory.

The next example pertains to the gravity/gauge theory duality. As we noted above, LQG starts by reformulating GR in terms of Yang-Mills type variables and making heavy use of gauge theory notions such as holonomies, Wilson loops and quantized fluxes. The recent finiteness results in supergravity rely heavily on the relation between structures that arise in the $\mathrm{N}=4$ super Yang-Mills theory and $\mathrm{N}=8$ supergravity in 4 dimensions. In the AdS/CFT correspondence this interplay is also at the forefront; for example, string theory in

${ }^{11}$ It is interesting, indeed, that the best non-perturbative definition of string theory takes us back to a local QFT, albeit on the boundary of spacetime. 
a 5 dimensional asymptotically AdS background is dual to this super Yang-Mills theory (on the boundary). Is this interplay between gravity and gauge theories a beacon guiding us to a rapprochement of various approaches? Could a deeper understanding of this duality lead to a new principle which has eluded us because we have examined the issue only piecemeal, from the perspective of only one approach at a time?

The last example is provided by the analyses of the statistical mechanical origin of black hole entropy. In string theory calculations one generally considers strings with end points of branes that carry gauge fields. In LQG, the horizon 'membrane' is pierced by the polymer excitations of the bulk quantum geometry and intrinsic geometry of quantum horizons is described by a gauge theory. Qualitatively, the two pictures appear to be similar. Yet, the detailed analyses are very different and their strengths and limitations are complementary. The string theory calculations refer to certain extremal and near extremal black holes, while in LQG they are based on the notion of isolated horizons and therefore include all black hole and cosmological horizons. But whereas the relation to semi-classical calculations of entropy is well understood in string theory, the issue remains open in LQG because what one counts is the number of microstates of the quantum horizon geometry which are conceptually quite distinct from the quantum corrections to the Euclidean, classical action. Given the qualitative similarity of concepts underlying these calculations, can one perhaps relate them in detail? Such a bridge would enable one to export the strengths of each of these calculations to overcome the corresponding limitation of the other. Thus, a better understanding of common elements, differences and relations between different approaches could well suggest a paradigm that combines deep ideas from various approaches.

Over the past three decades, such rapprochements were hindered by periodic bouts of irrational exuberance which led individual communities to be certain that theirs was the only viable path and, by implication, nothing else was worth paying attention to. To paraphrase the biologist François Jacob, for sustained progress in any area of science, it is important that the practitioners be aware of the limits of their science and thus their knowledge. Otherwise it is easy to mix what one believes and what one knows to create a misplaced sense of certitude. Ongoing dialogues across various approaches and careful examinations of the common elements and differences between them would go a long way to avoid this trap.

\section{Acknowledgments}

We have profited from discussions with a large number of colleagues, and correspondence with the authors of the 12 Chapters in this volume. This work was supported in part by the NSF grants PHY-1205388, PHY-0968612, PHY-1306441 and FRG-DMS-1263431, and the Eberly research funds of Penn state.

[1] A. Eddington, Report on the relativity theory of gravitation. (Fleetway Press, London, 1918); Report to the Physical Society of London, 1Reprinted 1920.

[2] G. Harvey, Observatory 99, 1958 (1979).

[3] S. W. Hawking and G. F. R. Ellis, The large scale structure of space-time (Cambridge University Press, Cambridge, 1973).

[4] H. Bondi, Cosmology (Cambridge University Press, Cambridge, 1960) 
[5] A. Taub, Ann. Math. 53, 472 (1951); Reprinted, with editorial introduction by M.A.H. MacCallum, in Gen. Rel. Grav. 36, 2689-2719 (2004).

[6] A. Petrov, Scientific Proceedings of Kazan State University (named after V.I. UlyanovLenin), Jubilee (1804-1954) Collection, 114, 5569 (1954). Translation by J. Jezierski and M.A.H. MacCallum, with introduction by M.A.H. MacCallum, Gen. Rel. Grav. 32, 16611685 (2000).

[7] H. Stephani, et al. Exact solutions of Einstein's field equations, 2nd edition, (Cambridge University Press, Cambridge 2003); Corrected Paperback edition, (2009).

[8] J. Griffiths and J Podolsky, Exact space-times in Einstein's general relativity, Cambridge Monographs in Mathematical Physics, (Cambridge University Press, Cambridge, 2009).

[9] V. Brumberg, Essential relativistic celestial mechanics (Adam Hilger, Bristol 1991).

[10] D. Lynden-Bell, Nature 22, 690694 (1969).

[11] H. Bondi, F. Pirani, and I. Robinson, Proc. Roy. Soc. Lond. A251, 519 (1959).

[12] K. Hurley, et al. [LIGO collaboration], Astrophys. J. 681, 14191428 (2008).

[13] A. H. Guth, Phys. Rev. D23, 347356 (1981).

[14] M. Harwit, Cosmic Discovery: The Search, Scope and Heritage of Astronomy (The MIT Press, Cambridge 1984).

[15] E. Kruse and E Agol, Science 344, 275 (2014).

[16] P. Coles and G. F. R. Ellis, Is the universe open or closed? The density of matter in the universe Cambridge Lecture Notes in Physics, vol. 7. (Cambridge University Press, Cambridge 1997).

[17] A. Einstein, and N. Rosen, Journal of Franklin Institute 223, 43-54 (1937).

[18] D. Kennefick, Physics Today 58, 43 (2005).

[19] H. Bondi, Nature 179, 1072-1073 (1957).

[20] F. A. E. Pirani, Phys. Rev. 105, 1089-1099 (1957).

[21] H. Bondi, F. A. E. Pirani and I. Robinson, Proc. Roy. Soc. Lond. A251, 519 (1959).

[22] R. A. Isaacson, Phys. Rev. 166, 1263 (1968).

[23] R. A. Isaacson, Phys. Rev. 166, 1272 (1968).

[24] K. Schwarzschild, Sitzber. Deut. Akad. Wiss. Berlin, Kl. Math-Phys. Tech. 189 (1916).

[25] R. P. Kerr, Phys. Rev. Lett., 11, 237 (1963).

[26] J. R. Oppenheimer, and G. M. Volkoff, Phys. Rev. 55, 374 (1939).

[27] J. R. Oppenheimer and H. Snyder, Phys. Rev. 56, 455 (1939).

[28] M. M. May and R. H. White, Phys. Rev. 141, 1232 (1966).

[29] D. Kennefick, Traveling at the Speed of Thought: Einstein and the Quest for Gravitational Waves, (Princeton University Press, Princeton 2007).

[30] J. Weber and J. A. Wheeler, Rev. Mod. Phys. 29, 509-515 (1957).

[31] J. Weber, Phys. Rev. Lett. 18, 498 (1967).

[32] J. Weber, Phys. Rev. Lett. 12, 1320-1324 (1969).

[33] P. Saulson, In: Receiving Gravitational Waves, (World Scientific, Singapore 2005), Chap. 9.

[34] O. D. Aguiar, Res. Astron. Astrophys. 11, 1-42 (2011).

[35] J. Levine and R. Stebbins, Phys. Rev. D 6, 1465-1468 (1972).

[36] J. J. Giganti, J. V. Larson, J. P. Richard, R. L. Tobias, and J. Weber, Lunar Surface Gravimeter Experiment Final Report (1977). See http://www.lpi.usra.edu/lunar/missions/apollo/apollo_17/experiments/lsg//.

[37] J. W. Armstrong, Living Rev. Relativity 9, 1 (2006).

[38] P. Saulson, Fundamentals of Interferometric Gravitational Wave Detectors, (World Scientific, 
Singapore (1994).

[39] V. B. Braginsky, Yu. I. Vorontsov, and F. Ya. Khalili, Sov. Phys. JETP 46, 705 (1977).

[40] C. Caves, Phys. Rev. D. 23, 1693-1708 (1981).

[41] R. Schnabel et al. Nature Physics Letter 7, 962-965 (2011).

[42] J. Aasi et al, Nature Photonics Letter 7, 613-619 (2013).

[43] L. L. Smarr, Gauge Conditions, Radiation Formulae and the Two Black Hole Collision, (Cambridge University Press, Cambridge 1979), Page 245.

[44] R. A. Hulse and J. H. Taylor, Astrophys. J. Lett., 195, L51 (1975).

[45] J. Abadie et al, Class. Quant. Grav. 27, 173001 (2010).

[46] S. W. Hawking, Phys. Rev. Lett. 26, 1344-1346 (1971).

[47] F. Pretorius, Phys. Rev. Lett. 95, 121101 (2005).

[48] J. G. Baker, J. Centrella, D. Choi, M. Koppitz, and J. van Meter, Phys. Rev. Lett. 96, 111102 (2006).

[49] M. Campanelli, C. Lousto, P. Marronetti and Y. Zlochower, Phys. Rev. Lett. 96, 111101 (2006).

[50] B. Allen et al, Phys. Rev. Lett. 83, 1498 (1999).

[51] J. Abadie et al, Sensitivity Achieved by the LIGO and Virgo Gravitational Wave Detectors during LIGO's Sixth and Virgo's Second and Third Science Runs, arXiv:1203.2674 [gr-qc] (2012).

[52] P. Astone et al, Phys. Rev. D 82, 022003 (2010).

[53] B. Abbott, et al, Phys. Rev. D 76, 022001 (2007).

[54] G. Hobbs et al, Class. Quantum Grav. 27, 084013 (2010).

[55] P. A. R. Ade et al, Phys. Rev. Lett. 112, 241101 (2014).

[56] B. S. Sathyaprakash and B. F. Schutz, Living Rev. Relativity 12, 2 (2009).

[57] C. M. Will, Living Rev. Relativity 17, 4 (2014).

[58] M. Punturo et al, Class. Quantum Grav. 27, 194002 (2010).

[59] Y. Choquet-Bruhat, Acta Math. 88 141-225 (1952).

[60] A. Lichnerowicz, J. Math. Pures Appl. 23 37-63 (1944).

[61] J. York, J. Math. Phys. 13 125-130 (1972).

[62] M. Holst, G. Nagy and G. Tsogtgerel, Comm. Math. Phys. 288 549-613 (2009).

[63] D. Maxwell, Comm. Math. Phys. 302 697-736 (2011).

[64] J. York, Phys. Rev. Lett. 82 1350-1353 (1999).

[65] D. Maxwell, unpub. gr-qc 1402.5585.

[66] D. Maxwell, unpub. gr-qc 1407.1467.

[67] J. Isenberg, R. Mazzeo and D. Pollack, Comm. Math. Phys. 231 529-568 (2002).

[68] P. Chrusciel, J. Isenberg and D. Pollack, Comm. Math. Phys. 257 29-42 (2005).

[69] J. Corvino, Comm. Math. Phys. 214 137-189 (2000).

[70] J. Corvino and R. Schoen, J. Diff. Geom. 73 185-217 (2006).

[71] A. Carlotto and R. Schoen, unpub. math 1407.4766.

[72] P. Chrusciel, J. Corvino and J. Isenberg, Comm. Math.Phys. 304 637-647 (2011).

[73] R. Bartnik, J. Diff. Geom. 37 31-71, (1993).

[74] R. Bartnik and J. Isenberg Class. Qtm. Grav. 23 2559-2569 (2006).

[75] R. Arnowitt, S. Deser and C. Misner, Phys. Rev. 122 997-1006 (1961).

[76] R. Schoen and S.-T. Yau, Comm. Math. Phys. 65 45-76 (1979).

[77] E. Witten, Comm. Math. Phys. 80 381-402 (1981)

[78] G. Huisken and T. Ilmanen, J. Diff. Geom. 59 353-437 (2001). 
[79] H. Bray, J. Diff. Geom. 59 177-267 (2001).

[80] M. Eichmair, L.-H. Huang, D. Lee and R. Schoen, unpub. math 1110.2087.

[81] R. Geroch, J. Math. Phys. 9 450-465 (1968).

[82] Y. Choquet-Bruhat and R. Geroch, Comm. Math. Phys. 14 329-335 (1968).

[83] J. Sbierski, unpub. gr-qc 1309.7591.

[84] V. Moncrief, Phys. Rev. D 23 312-315 (1981).

[85] H. Ringström, Ann. Math.170 1181-1240 (2009).

[86] D. Christodoulou and S. Klainerman, The Global Nonlinear Stability of Minkowski Space, Princeton U. Press (1994).

[87] D. Christodoulou, The Formation of Black Holes in General Relativity, EMS (2009).

[88] H. Friedrich, J. Geom. Phys. 3 101-117 (1986).

[89] L. Andersson and V. Moncrief, in Cauchy Problem in General Relativity, ed. P. Chrusciel and H. Friedrich, Birkahauser (2004).

[90] H. Ringström, Invent. Math. 173 123-208 (2008).

[91] H. Ringström, Comm. Math. Phys. 290 155-218 (2009).

[92] C. Svedberg, Ann H. Poinc. 12 849-917 (2011).

[93] X. Luo and J. Isenberg, Ann. Phys. 334 420-454 (2013).

[94] S. Klainerman, I. Rodnianski and J. Szeftel, unpub. gr-qc 1204.1772.

[95] D. Eardley and V. Moncrief, Comm. Math. Phys. 83, 171-212 (1982).

[96] F. Pretorius, Phys. Rev. Lett. 95121101 (2005).

[97] S. Hahn and R. Lindquist, Ann. Phys. 29 304-331 (1964).

[98] M. Campanelli et. al., Phys. Rev. Lett. 96111101 (2006).

[99] J. Baker et. al., Phys. Rev. Lett. 96111102 (2006).

[100] M. Choptuik, Phys. Rev. Lett. 70 9-12 (1994).

[101] B. Berger and V. Moncrief, Phys. Rev. D 48, 4676-4687 (1993).

[102] V. Belinskii, E. Lifshitz, and I. Khalatnikov, Zh. Eksp. Teor. Fiz. 62, 1606-1613 (1972).

[103] H. Ringström, Ann. Math. 170 1181-1240 (2009).

[104] P. Bizon, Gen. Rel. Grav. 461724 (2014).

[105] L. Lehner and F. Pretorius, Phys. Rev. Lett. 105101102 (2010).

[106] S. A. Hayward, gr-qc/0008071.

[107] A. Ashtekar et al, Phys. Rev. Lett. 85, 3564-3567 (2000).

[108] A. Ashtekar and B. Krishnan, Living Rev. Relativity 710 (2004).

[109] E. Gourgoulhon, J. L. Jaramillo, Phys. Rept. 423 159-294 (2006).

[110] A. Ashtekar and M. Campiglia, Phys. Rev. D88, 064045 (2013).

[111] R. Owen, Phys. Rev. D80, 084012 (2009).

[112] J. M. Bardeen, B. Carter and S. W. Hawking, Commun. Math. Phys. 31, 161-170 (1973).

[113] Black Holes, Les Houches 1972, C. DeWitt and B. DeWitt eds (Gordon and Breach, New York, 1973).

[114] A. Ashtekar, F. Pretorius and F. M. Ramazanoglu, Phys. Rev. Lett. 106161303 (2011); Phys. rev. D83, 044040 (2011).

[115] J. M. M Senovilla, In Relativity and Gravitation: 100 Years after Einstein in Prague, J. Bicak and T. Ledvinka, eds (Springer, Berlin, 2014).

[116] A. Ashtekar and G. J. Galloway, Adv. Theor. Math. Phys. 9 1-30 (2005).

[117] Proceedings on theory of gravitation, I. Infeld ed (Guthier Villars, Paris and PWN Editions Scientific de Pologne, Warszawa (1964)).

[118] A. Einstein, Meaning of Relativity (Princeton University Press, Princeton, 1945). 
[119] L. Rosenfeld, Ann. Physik 5, 113 (1930); Z. Physik 65587 (1930).

[120] M. P. Bronstein, Phys. Zeitschr. der Sowjetunion 9, 140-157 (1936);

S. Deser and A. Starobinski, Gen. Rel. Grav. 44 263-265 (2012).

[121] M. P. Bronstein, Zh.ETF (JETP) 6195 (1936);

D. J. Cirlio-Lombardo, arXiv:1405.2334.

[122] R. P. Feynman, Acta Physica Polonica, 24, 697-722 (1964).

[123] B. S. DeWitt, in Magic without Magic: John Archibald Wheeler edited by J. R. Klauder (W. H. Freeman, San Fransico 1972).

[124] G. 't Hooft and M. J. G. Veltman, Annales Poincaré Phys. Theor. A20 69 (1974).

[125] M. H. Goroff and A. Sagnotti, Nucl. Phys. B266 709 (1986).

[126] K. S. Stelle, Phys. Rev. D16 953 (1977);

T. Tomboulis, Phys. Lett. B97 77 (1980).

[127] S. Deser, J. Kay and K. S. Stelle, Phys. Rev. Lett. 38, 527 (1977);

M. T. Grisaru, P. Van Nieuwenhuizen and J. A. M. Vermaseren, Phys. Rev. Lett. 37, 1662 (1976).

[128] P. S. Howe and K. S. Stelle, Int. J. Mod. Phys. A4, 1871 (1989).

[129] L. Parker, Ph. D. Dissertation (1967).

[130] R. Arnowitt, S. Deser and C. W. Misner, in Gravitation: An introduction to current research ed L. Witten (John Wiley, New York, 1962).

[131] A. Komar,in Relativity Carmeli M, Fickler S. I. and Witten L (eds) (Plenum, New York, 1080).

[132] P. G. Bergmann and A. Komar, General Relativity and Gravitation vol 1, On Hundred Years after the Birth of Albert Einstein, ed A. Held (Plenum, New York, 1980).

[133] A. Ashtekar and R. Geroch, Quantum theory of gravitation, Rep. Prog. Phys. 37 1211-1256 (1974).

[134] K. Kuchař, in Quantum Gravity 2, A Second Oxford Symposium ed C. J. Isham, R. Penrose and D. W. Sciama (Clarendon Press, Oxford, 1975).

[135] J. A. Wheeler,Geometrodynamics, (Academic Press, New York, 1964).

[136] J. A. Wheeler, in Relativity, Groupos and Topology eds DeWitt C M and DeWitt B S (Gordon and Breach, New York, 1963).

[137] S. Weinberg Gravitation and Cosmology (John Wiley, New York, 1972).

[138] L. H. Ford and C. H. Wu, AIP Conf. Proc. 977 145-159 (2008).

[139] A. Everett and T. Roman, Time Travel and Warp Drives: A Scientific Guide to Shortcuts through Time and Space (U of Chicago, Chicago, 2012).

[140] C. P. Burgess, Living Rev. Relativity 75 (2004).

[141] R. A. Porto and I. Z. Rothstein, Phys. Rev. D78 044013 (2008), D81 029902 (2010).

[142] A. Ashtekar, Phys. Rev. Lett. 46, 573 (1981); J. Math. Phys. 222885 (1987); Asymptotic Quantization (Bibliopolis, Naples, 1987);

A. Strominger, arXiv:1312.2229

T. He, V. Lysov, P. Mitra and A. Strominger, arXiv:1401.7026.

[143] L. Mason and D. Skinner, arXiv:0808.3907;

F. Cachazo and Y. Geyer, arXiv:1206.6511;

F. Cachazo, S. He, and Y. E. Yuan., arXiv:1307.2199;

N. Arkani-Hamed and J. Trnka, arXiv:1312.2007.

[144] R. Penrose and W. Rindler, Spinors and Space-Time: Volume 2, Spinor and Twistor Methods in Space-Time Geometry (Cambridge University Press, Cambridge 1988). 
[145] H. W. Hamber, Quantum gravitation: The Feynman path integral approach (Springer, Berlin (2009)).

[146] P. Hořava, Phys. Rev. D79 084008 (2009).

[147] F. Dowker, Gen. Rel. Grav. 45, 1651-1667 (2013).

[148] M. Vassiliev, Introduction to Higher Spin Gauge Theory, Kramer's course, (University of Utretcht, Utretcht (2014)).

[149] Some Strangeness in Proportion, H. Wolf, ed. (Addison Wesley, Reading, 1980).

[150] General Relativity, an Einstein Centennial survey S. W. Hawking and W. Israel, eds. (Cambridge University Press, Cambridge, 1979).

[151] S. W. Hawking, Is the End In Sight for Theoretical Physics?: An Inaugural Address (Cambridge University Press, Cambridge, 1980).

[152] S. Carlip, arXiv:1207.4503.

[153] H. Nicolai, Physics 2, 70 (2009); Personal communication (2014).

[154] D. J. Gross and V. Periwal, Phys. Rev. Lett. 601517 (1988). 